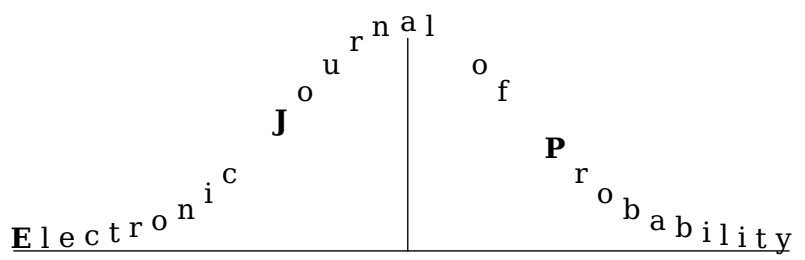

Electron. J. Probab. 26 (2021), article no. 31, 1-22.

ISSN: 1083-6489 https://doi.org/10.1214/20-EJP569

\title{
Thin times and random times' decomposition
}

\author{
Anna Aksamit* $\quad$ Tahir Choulli $^{\dagger} \quad$ Monique Jeanblanc $^{\ddagger}$
}

\begin{abstract}
The paper defines and studies thin times which are random times whose graph is contained in a countable union of graphs of stopping times with respect to a reference filtration $\mathbb{F}$. We show that a generic random time can be decomposed into thin and thick parts, where the second is a random time avoiding all $\mathbb{F}$-stopping times. Then, for a given random time $\tau$, we introduce $\mathbb{F}^{\tau}$, the smallest right-continuous filtration containing $\mathbb{F}$ and making $\tau$ a stopping time, and we show that, for a thin time $\tau$, each $\mathbb{F}$-martingale is an $\mathbb{F}^{\tau}$-semimartingale, i.e., the hypothesis $\left(\mathcal{H}^{\prime}\right)$ for $\left(\mathbb{F}, \mathbb{F}^{\tau}\right)$ holds. We present applications to honest times, which can be seen as last passage times, showing classes of filtrations which can only support thin honest times, or can accommodate thick honest times as well.
\end{abstract}

Keywords: thin times; graph of a random time; avoidance of stopping time; dual optional projection; progressive enlargement of filtration; (semi)martingale stability; hypothesis $\left(\mathcal{H}^{\prime}\right)$; honest times; thin-thick decomposition; immersion.

MSC2020 subject classifications: 60G07; 60G40; 60G44.

Submitted to EJP on March 5, 2020, final version accepted on December 6, 2020.

\section{Introduction}

The paper studies the class of thin times in an enlargement of filtration framework. The concept naturally fits, and complements, the studies of random times and progressive enlargement of filtrations. A random time defined on a filtered probability space $(\Omega, \mathcal{G}, \mathbb{F}, \mathbb{P})$ with $\mathbb{F}=\left(\mathcal{F}_{t}\right)_{t \geq 0}$, is a random variable with values in $[0, \infty]$. In the literature of enlargement on filtration, e.g., Mansuy and Yor [21] and Nikeghbali [23], it is common to assume that the random time $\tau$ avoids all $\mathbb{F}$-stopping times, i.e., $\mathbb{P}(\tau=T<\infty)=0$ for any $\mathbb{F}$-stopping time $T$. The motivation behind our work is to explore what happens if this condition fails. In Definition 1.1 we introduce thin times which satisfy the opposite

*School of Mathematics and Statistics, University of Sydney, Australia.

E-mail: anna.aksamit@sydney.edu.au

${ }^{\dagger}$ Mathematical and Statistical Sciences Department, University of Alberta, Canada.

E-mail: tchoulli@ualberta.ca

${ }^{\ddagger}$ Laboratoire de Mathématiques et Modélisation d’Évry (LaMME), UMR CNRS 8071, Université d’Évry-Vald’Essonne, Université Paris Saclay, France.

E-mail: monique.jeanblanc@univ-evry.fr 
property, i.e., their graph is contained in a countable union of graphs of $\mathbb{F}$-stopping times. We emphasise that in the discrete time set-up all stopping times, and random times in general, have countably many values and hence are thin. That is yet another natural motivation to study this class in general continuous time set-up. The given name is motivated by the fact that the graph of a thin random time is contained in a thin set (see [13, Chapter I, Definition 1.30] for definition and main properties of thin sets). The notion of thin time was mentioned, but not developed, for the first time in Dellacherie and Meyer [7] under the name arlequine random variable referring to the costume of the Harlequin which is made of patches of different colors. On the other hand, we also work with thick times which are introduced in Definition 2.1, and satisfy the above avoidance condition, and the graph of a thick random time does not intersect any thin set (i.e., the intersection is an evanescent set). In Section 1, we show the first results on thin times. Our study strongly relies on the notion of dual optional projection and other processes linked to the general theory of stochastic processes, in particular, to the enlargement of filtration theory.

Since their introduction in the 1980's, enlargements of filtrations have remained an important tool and field of study in the theory of stochastic processes. In fact the theory has seen its second youth recently with revised interest sparked by applications in mathematical finance. These include, in particular, credit risk and modelling of asymmetry of information, where one considers a financial market where different agents have different levels of information.

Enlargement of filtration theory, to which we contribute here, focuses on the properties of stochastic processes under a change of filtration. The behaviour of (semi)martingales under a suitable change of filtration may be seen as parallel to absolutely continuous change of measure and Girsanov's theorem (see [12, 27, 28]). It is of a fundamental interest to provide new classes of enlargements under which the semimartingale property is stable.

Thin times form a new class of random times which possesses this property under progressive enlargement. Recall that for a random time $\tau, \mathbb{F}^{\tau}:=\left(\mathcal{F}_{t}^{\tau}\right)_{t \geq 0}$ denotes the right-continuous filtration $\mathbb{F}$ progressively enlarged with $\tau$, and is given by

$$
\mathcal{F}_{t}^{\tau}:=\bigcap_{s>t}\left(\mathcal{F}_{s} \vee \sigma(\tau \wedge s)\right) \quad \text { for any } t \geq 0 .
$$

The fundamental question in the enlargement of filtration theory is if all $\mathbb{F}$-martingales remain $\mathbb{F}^{\tau}$-semimartingales. If the latter property is satisfied we say, as it is done in the literature, that the hypothesis $\left(\mathcal{H}^{\prime}\right)$ holds for $\left(\mathbb{F}, \mathbb{F}^{\tau}\right)$, in which case we are interested in the $\mathbb{F}^{\tau}$-semimartingale decomposition of $\mathbb{F}$-martingales (if $\left(\mathcal{H}^{\prime}\right)$ holds for $\left(\mathbb{F}, \mathbb{F}^{\tau}\right), \mathbb{F}$ martingales are necessary $\mathbb{F}^{\tau}$-special semimartingales). The main result in Subsection 4.1 is Theorem 4.1 where we establish the hypothesis $\left(\mathcal{H}^{\prime}\right)$ for thin random times and give the corresponding semimartingale decomposition.

In Section 2, we define the decomposition of a random time into thick and thin parts which we call the thin-thick decomposition. The thin-thick decomposition is congruent with the decomposition of a stopping time into accessible and totally inaccessible parts. One of the main results in this section, Theorem 2.5, says that any random time $\tau$ admits a unique thin-thick decomposition and characterizes its thin and thick components in terms of the dual optional projection of the indicator process $\mathbb{1}_{\llbracket \tau, \infty}$. In Section 2, we also show the significance of thin-thick decomposition for the hypothesis $\left(\mathcal{H}^{\prime}\right)$ and immersion in the context of the progressive enlargement of filtration.

In Section 3, we turn to honest times which constitute an important and well studied class of random times (see Barlow [3] and Jeulin [17]) and can be suitably represented as last passage times. Adopting the notion of jumping filtration from Jacod and Skorokhod 
[14] we show in Theorem 3.6, which is the main result of this section, that such a filtration can only support honest times which are thin. That includes the compound Poisson process filtration. In [14], the link between jumping filtration and finite variation martingales is established; further developments related to purely discontinuous martingale filtrations are presented in Hannig [10]. In Theorem 3.6, we also show that there exists a thick honest time in any filtration which can accommodate a non-constant continuous martingale. In Section 3, we also discuss two examples of thin honest times: the last passage time at a barrier $a$ of a compound Poisson process and an example based on an approximation of a Brownian local time.

\section{A definition and some properties of thin times}

\subsection{New class of random times}

Let $(\Omega, \mathcal{G}, \mathbb{P})$ be a complete probability space, equipped with a filtration $\mathbb{F}:=\left(\mathcal{F}_{t}\right)_{t \geq 0}$ satisfying the usual conditions of completeness and right-continuity, and such that $\mathcal{F}_{\infty}:=\bigcup_{t>0} \mathcal{F}_{t} \subset \mathcal{G}$. For any càdlàg process $X$ we denote by $X_{-}$the left-continuous version of $X$, by $\Delta X$ the jump of $X$ and by $X_{\infty}$ the $\operatorname{limit}_{t \rightarrow \infty} X_{t}$ if it exists. The process $X$ is said to be increasing if, for almost all $\omega$, it satisfies $X_{t}(\omega) \geq X_{s}(\omega)$ for all $t \geq s$. A random variable is said to be positive if it has values in $[0, \infty)$. We denote by $G \cdot X$ the stochastic integral of a predictable process $G$ w.r.t. a semimartingale $X$, when this integral is well defined.

Consider a random time $\tau$, i.e., a $[0, \infty]$-valued $\mathcal{G}$-measurable random variable. Note that a random time $\tau$ is not necessarily $\mathcal{F}_{\infty}$-measurable. For a random time $\tau$, we denote by $\llbracket \tau \rrbracket:=\left\{(\omega, t) \subset \Omega \times \mathbb{R}^{+}: \tau(\omega)=t\right\}$ its graph. Let us recall, following [17], some useful processes associated with the pair $(\mathbb{F}, \tau)$. For the process $A:=\mathbb{1}_{\llbracket \tau, \infty \mathbb{I}}$, we denote by $A^{p}$ its $\mathbb{F}$-dual predictable projection and by $A^{o}$ its $\mathbb{F}$-dual optional projection (for reader's convenience definitions are recalled in Appendix A). By an abuse of language, $A^{o}$ is also called the dual optional projection of the random time $\tau$. We also define two F-supermartingales $Z$ and $\widetilde{Z}$ as the optional projections of processes $1-A$ and $1-A_{-}$ respectively, i.e.,

$$
Z_{t}:=o\left[\mathbb{1}_{\llbracket 0, \tau \llbracket}\right]_{t}=\mathbb{P}\left(\tau>t \mid \mathcal{F}_{t}\right) \quad \text { and } \quad \widetilde{Z}_{t}:=o\left[\mathbb{1}_{\llbracket 0, \tau \rrbracket}\right]_{t}=\mathbb{P}\left(\tau \geq t \mid \mathcal{F}_{t}\right) \text {. }
$$

Since the dual optional projection $A^{o}$ will play a crucial role in the paper, we recall two equalities where it appears (see [17, Chapitre IV, section 1]):

$$
A^{o}=m-Z \text { and } \Delta A^{o}=\widetilde{Z}-Z,
$$

where $m$ is a BMO $\mathbb{F}$-martingale. Furthermore, $\widetilde{Z}=Z_{-}+\Delta m$.

The following definition contains the leading idea of the paper. It introduces a class of random times using a criterion based on $\mathbb{F}$-stopping times w.r.t. a reference filtration.

Definition 1.1. A random time $\tau$ is called an $\mathbb{F}$-thin time if its graph $\llbracket \tau \rrbracket$ is contained in an $\mathbb{F}$-thin set, i.e., if there exists a sequence of $\mathbb{F}$-stopping times $\left(T_{n}\right)_{n=1}^{\infty}$ with disjoint graphs such that $\llbracket \tau \rrbracket \subset \bigcup_{n=1}^{\infty} \llbracket T_{n} \rrbracket$. Moreover,

(a) Let $T_{0}:=\infty$. We say that the sequence $\left(T_{n}\right)_{n \geq 0}$ exhausts the $\mathbb{F}$-thin time $\tau$ or that $\left(T_{n}\right)_{n \geq 0}$ is an $\mathbb{F}$-exhausting sequence of the $\mathbb{F}$-thin time $\tau$.

(b) We say that the family of sets $\left(C_{n}\right)_{n \geq 0}$, given by $C_{0}:=\{\tau=\infty\}$ and $C_{n}:=\left\{\tau=T_{n}<\right.$ $\infty$ \} for $n \geq 1$, is an $\mathbb{F}$-partition of the $\mathbb{F}$-thin time $\tau$.

(c) We say that the family of bounded càdlàg $\mathbb{F}$-martingales $\left(z^{n}\right)_{n \geq 0}$ given by their terminal values $\mathbb{P}\left(C_{n} \mid \mathcal{F}_{\infty}\right)$, namely $z_{t}^{n}:=\mathbb{P}\left(C_{n} \mid \mathcal{F}_{t}\right)$, is a martingale family of the thin time $\tau$. 
If this is clear from the context, we shall simply say that $\tau$ is a thin time instead of saying that $\tau$ is an $\mathbb{F}$-thin time, etc. Note that a thin time $\tau$ is built from $\mathbb{F}$-stopping times, i.e., $\tau=\sum_{n>0} T_{n} \mathbb{1}_{C_{n}}$ where $\left(T_{n}\right)_{n \geq 0}$ is one exhausting sequence and $\left(C_{n}\right)_{n \geq 0}$ is its partition. On the other hand, given a sequence $\left(T_{n}\right)_{n \geq 0}$ of $\mathbb{F}$-stopping times with disjoint graphs such that $T_{0}=\infty$ and a partition $\left(C_{n}\right)_{n \geq 0}$ of $\Omega$, the random time $\tau$ defined as $\tau:=\infty \mathbb{1}_{C_{0}}+\sum_{n \geq 1} T_{n} \mathbb{1}_{C_{n}}$ is thin.

Let us also remark that an exhausting sequence $\left(T_{n}\right)_{n \geq 0}$ of a thin time is not unique, however the properties of a thin time do not depend on the specific choice of an exhausting sequence. The following proposition combines two exhausting sequences of a given thin time.

Proposition 1.2. Let $\tau$ be a thin time with an exhausting sequence $\left(T_{n}\right)_{n \geq 0}$ and a partition $\left(C_{n}\right)_{n \geq 0}$. Suppose that $\left(S_{n}\right)_{n \geq 0}$ and $\left(B_{n}\right)_{n \geq 0}$ are as well an exhausting sequence and a partition of $\tau$. Then, $\left(U_{0},\left(U_{n, m}\right)_{n \geq 1, m \geq 1}\right)$, defined as $U_{0}:=\infty$ and $U_{n, m}:=T_{n} \mathbb{1}_{\left\{T_{n}=S_{m}\right\}}+\infty \mathbb{1}_{\left\{T_{n} \neq S_{m}\right\}}$ for $n \geq 1$ and $m \geq 1$, is an exhausting sequence of $\tau$ and $\left(D_{0},\left(D_{n, m}\right)_{n \geq 1, m \geq 1}\right)$ defined as $D_{0}:=\{\tau=\infty\}$ and $D_{n, m}:=C_{n} \cap B_{m}$ for $n \geq 1$ and $m \geq 1$, is the corresponding partition of $\tau$.

Proof. Firstly note that $U_{n, m}$ is a stopping time for any pair $n \geq 1$ and $m \geq 1$ since $\left\{T_{n}=S_{m}\right\} \in \mathcal{F}_{T_{n} \wedge S_{m}}$. Secondly note that the following identity holds:

$$
\tau=\infty \mathbb{1}_{\{\tau=\infty\}}+\sum_{n \geq 1} \sum_{m \geq 1} T_{n} \mathbb{1}_{\left\{\tau=T_{n}=S_{m}<\infty\right\}}=\infty \mathbb{1}_{D_{0}}+\sum_{n \geq 1} \sum_{m \geq 1} U_{n, m} \mathbb{1}_{\left\{\tau=U_{n, m}<\infty\right\}} .
$$

Hence it remains to show that $\left(U_{n, m}\right)_{n \geq 1, m \geq 1}$ have disjoint graphs which follows by observing that the sets

$$
\llbracket U_{n, m} \rrbracket \cap \llbracket U_{k, l} \rrbracket \subset \llbracket T_{n} \rrbracket \cap \llbracket T_{k} \rrbracket \text { and } \llbracket U_{n, m} \rrbracket \cap \llbracket U_{k, l} \rrbracket \subset \llbracket S_{m} \rrbracket \cap \llbracket S_{l} \rrbracket
$$

are evanescent if $n \neq k$ or $m \neq l$.

Thin times, unlike other classes of random times, possess many stability properties as those described in the following remark.

Remark 1.3. (a) Let $\mathbb{Q}$ be a probability measure absolutely continuous w.r.t. $\mathbb{P}$ and $\widetilde{\mathbb{F}}$ be the filtration $\mathbb{F}$ completed with $\mathbb{Q}$-null sets. Then an $\mathbb{F}$-thin time is an $\widetilde{\mathbb{F}}$-thin time since $\mathbb{F} \subset \widetilde{\mathbb{F}}$. In other words thin times are invariant w.r.t. an absolutely continuous change of measure.

(b) Let $\mathbb{G}$ be a filtration such that $\mathbb{F} \subset \mathbb{G}$. Then any $\mathbb{F}$-thin time is a $\mathbb{G}$-thin time since any $\mathbb{F}$-stopping time is a $\mathbb{G}$-stopping time. In other words, thin times are stable under filtration enlargement.

(c) Let $\tau$ and $\sigma$ be two $\mathbb{F}$-thin times with exhausting sequences $\left(T_{n}\right)_{n \geq 0}$ and $\left(S_{n}\right)_{n \geq 0}$ respectively. Then $\tau \wedge \sigma$ and $\tau \vee \sigma$ are also $\mathbb{F}$-thin times since

$$
\llbracket \tau \wedge \sigma \rrbracket \subset \bigcup_{n \geq 1} \llbracket T_{n} \rrbracket \cup \bigcup_{n \geq 1} \llbracket S_{n} \rrbracket \text { and } \llbracket \tau \vee \sigma \rrbracket \subset \bigcup_{n \geq 1} \llbracket T_{n} \rrbracket \cup \bigcup_{n \geq 1} \llbracket S_{n} \rrbracket .
$$

The following theorem provides a useful characterization of a thin time based on its F-dual optional projection.

Theorem 1.4. A random time is a thin time if and only if its dual optional projection is a pure jump process.

Proof. For any sequence $\left(S_{n}\right)_{n \geq 1}$ of $\mathbb{F}$-stopping times with disjoint graphs, we have

$$
\sum_{n=1}^{\infty} \mathbb{P}\left(\tau=S_{n}<\infty\right)=\sum_{n=1}^{\infty} \mathbb{E}\left[\Delta A_{S_{n}}^{o} \mathbb{1}_{\left\{S_{n}<\infty\right\}}\right]
$$


as, from definition of dual optional projection, $\mathbb{P}(\tau=S<\infty)=\mathbb{E}\left[\Delta A_{S}^{o} \mathbb{1}_{\{S<\infty\}}\right]$ for any stopping time $S$. Since also by definition of the dual optional projection $\mathbb{E}\left[A_{\infty}^{o}\right]=$ $\mathbb{P}(\tau<\infty)$, and using the fact that $A^{o}$ is an increasing process, we conclude that the sequence $\left(T_{n}\right)_{n>0}$ with $T_{0}=\infty$ is an exhausting sequence of $\tau$, i.e., satisfies the condition $\sum_{n=1}^{\infty} \mathbb{P}\left(\tau=T_{n}<\infty\right)=\mathbb{P}(\tau<\infty)$, if and only if it satisfies the condition $\mathbb{E}\left[A_{\infty}^{o}\right]=\sum_{n=1}^{\infty} \mathbb{E}\left[\Delta A_{T_{n}}^{o} \mathbb{1}_{\left\{T_{n}<\infty\right\}}\right]$. In other words, $\tau$ is a thin time if and only if $A^{o}$ is a pure jump process.

The following result describes how, after a thin time, the conditional expectations with respect to elements of $\mathbb{F}^{\tau}$ can be expressed in terms of the conditional expectations with respect to elements of $\mathbb{F}$. For an arbitrary random time, one is able to express $\mathbb{F}^{\tau}$-conditional expectations in terms of $\mathbb{F}$-conditional expectations only strictly before $\tau$ (this result is often referred to as key lemma in enlargement of filtration literature, see Lemma 3.1 in [9] and Section 3.1.1 in [4]). A powerful property of thin times is that one can obtain this kind of result also after $\tau$ as described below. It is crucial for results in Section 4.1.

The proof of the following Lemma 1.5 is given in Appendix C.

Lemma 1.5. Let $\tau$ be a thin time with exhausting sequence $\left(T_{n}\right)_{n \geq 0}$, partition $\left(C_{n}\right)_{n \geq 0}$ and martingale family $\left(z^{n}\right)_{n \geq 0}$. Then:

(a) $z_{t}^{n}>0$ and $z_{t-}^{n}>0$ for all $t \geq 0$ a.s. on $C_{n}$ for each $n \geq 0$.

(b) The progressive enlargement of filtration $\mathbb{F}$ with $\tau, \mathbb{F}^{\tau}:=\left(\mathcal{F}_{t}^{\tau}\right)_{t \geq 0}$, defined by $\mathcal{F}_{t}^{\tau}:=\bigcap_{u>t} \mathcal{F}_{u} \vee \sigma(\{\tau \leq s\}: s \leq u\}$, satisfies

$$
\mathcal{F}_{t}^{\tau}=\bigcap_{u>t} \mathcal{F}_{u} \vee \sigma\left(C_{n} \cap\left\{T_{n} \leq s\right\}, s \leq u, n \geq 1\right) .
$$

(c) For any $n \geq 1$ and any $\mathcal{G}$-measurable integrable random variable $X$, we have

$$
\mathbb{E}\left[X \mid \mathcal{F}_{t}^{\tau}\right] \mathbb{1}_{\left\{t \geq T_{n}\right\} \cap C_{n}}=\mathbb{1}_{\left\{t \geq T_{n}\right\} \cap C_{n}} \frac{\mathbb{E}\left[X \mathbb{1}_{C_{n}} \mid \mathcal{F}_{t}\right]}{z_{t}^{n}} .
$$

Corollary 1.6. It follows immediately that, for $s \leq t, n \geq 1$ and any $\mathcal{G}$-measurable integrable random variable $X$

$$
\mathbb{E}\left[X \mid \mathcal{F}_{t}^{\tau}\right] \mathbb{1}_{\left\{s \geq T_{n}\right\} \cap C_{n}}=\mathbb{1}_{\left\{s \geq T_{n}\right\} \cap C_{n}} \frac{\mathbb{E}\left[X \mathbb{1}_{C_{n}} \mid \mathcal{F}_{t}\right]}{z_{t}^{n}} .
$$

\subsection{Application to market incompleteness}

In Kardaras and Ruf [20], the authors, among other problems, study the question whether a complete market can become incomplete after shrinking the filtration. In Section 5.1 therein, the following motivating example is considered. Let $W$ be a Brownian motion and $\mathbb{F}^{W}$ be its natural filtration. Let $B$ be the Lévy transformation of $W$, i.e., $B=\int_{0}^{\cdot} \operatorname{sgn}\left(W_{s}\right) d W_{s}$, and $\mathbb{F}^{B}$ its natural filtration.

Then $\mathbb{F}^{B}=\mathbb{F}^{|W|} \subsetneq \mathbb{F}^{W}$ and the stochastic exponential $S=\mathcal{E}(B)$ has a predictable representation property in both $\mathbb{F}^{B}$ and $\mathbb{F}^{W}$, in particular $\left(S, \mathbb{F}^{W}\right)$-market is complete. The authors consider the $\mathbb{F}^{W}$-stopping time

$$
\tau:=\inf \left\{t \geq 0: W_{t}=1\right\},
$$

and note that $\tau$ is not $\mathbb{F}^{B}$-stopping time. The filtration $\mathbb{F}$ is defined as the progressive enlargement of $\mathbb{F}^{B}$ with $\tau$. Next, a sequence of $\mathbb{F}^{B}$-stopping times is defined by

$$
T_{n}:=\inf \left\{t>S_{n-1}:\left|W_{t}\right|=1\right\}, \quad \text { with } \quad S_{n}:=\inf \left\{t>T_{n}:\left|W_{t}\right|=0\right\} \quad \text { and } \quad S_{0}=0 .
$$


Then, the process

$$
N:=\mathbb{1}_{\llbracket \tau, \infty \mathbb{I}}-\frac{1}{2} \sum_{n=1}^{\infty} \mathbb{1}_{\llbracket T_{n}, \infty \mathbb{I}} \mathbb{1}_{\left\{\tau \geq T_{n}\right\}}
$$

is an $\mathbb{F}$-martingale but not an $\mathbb{F}^{W}$-local martingale (since it is not continuous). Hence, the $(S, \mathbb{F})$-market is not complete (as $S$ has no predictable representation property in $\mathbb{F}$ ).

We note here that in fact $\tau$ is an $\mathbb{F}^{B}$-thin time with exhausting sequence $\left(T_{n}\right)$, indeed $\llbracket \tau \rrbracket \subset \bigcup_{n=1}^{\infty} \llbracket T_{n} \rrbracket$. The random time $\tau$ is an $\mathbb{F}$-accessible stopping time since $T_{n}$ are already $\mathbb{F}^{B}$-predictable stopping times, and $\mathbb{F}$ is not a quasi left continuous filtration (compare also with Remark 2.8(b)). The above example from [20] works in analogous way for any $\mathbb{F}^{B}$-thin time $\tau$, and illustrates natural interest in this class of times.

We also remark that the above example illustrates well the result in [5, Proposition 9 , p.289]. The process $B$ is both an $\mathbb{F}$ and an $\mathbb{F}^{W}$-martingale which has predictable representation property in $\mathbb{F}^{W}$. However, $B$ has no predictable representation property in $\mathbb{F}$, and $\mathbb{F}$ is not immersed in $\mathbb{F}^{W}$.

\section{Thin-thick decomposition of a random time}

In this section we present an application of thin times to the decomposition of a generic random time into thin and thick parts. In the first subsection, we introduce and present some results about thick times. Then, in the second subsection, we establish the thin-thick decomposition. Finally, in the remaining subsections, we apply thin-thick decomposition to obtain results on the hypothesis $\left(\mathcal{H}^{\prime}\right)$ and immersion.

\subsection{Thick times}

As described in the introduction, thick times avoid stopping times from the reference filtration, i.e., thick times are defined in the following way.

Definition 2.1. A random time $\tau$ is called a thick time if $\llbracket \tau \rrbracket \cap \llbracket T \rrbracket$ is evanescent for any $\mathbb{F}$-stopping time $T$, i.e., if it avoids all $\mathbb{F}$-stopping times.

Similarly as for thin times in Theorem 1.4, thick times can be characterized in terms of their dual optional projection.

Theorem 2.2. A random time is a thick time if and only if its dual optional projection is a continuous process. In that case $A^{o}=A^{p}$.

Proof. Let $T$ be an $\mathbb{F}$-stopping time. Since $\mathbb{E}\left[\Delta A_{T}^{o} \mathbb{1}_{\{T<\infty\}}\right]=\mathbb{P}(\tau=T<\infty)$ and $A^{o}$ is an increasing process, we deduce that

$$
\mathbb{P}(\tau=T<\infty)=0 \quad \text { if and only if } \quad \Delta A_{T}^{o} \mathbb{1}_{\{T<\infty\}}=0 \quad \text { P-a.s. }
$$

Since $\left\{\Delta A^{o}>0\right\}$ is an optional set, the optional section theorem [11, Theorem 4.7] implies that $\left\{\Delta A^{o}>0\right\}$ is exhausted by disjoint graphs of $\mathbb{F}$-stopping times. Thus, we conclude that $\tau$ is a thick time if and only if $A^{o}$ is continuous.

The straightforward observation that the two classes of thin and thick times have trivial intersection is stated in the following obvious lemma.

Lemma 2.3. A random time $\tau$ belongs to the class of thick times and to the class of thin times if and only if $\tau=\infty$.

\subsection{Decomposition of a random time}

The main concept of this section, the thin-thick decomposition, is presented in the next definition. It is followed by the result stating the existence of such a decomposition for any random time. 
Definition 2.4. Consider a random time $\tau$. A pair of random times $\left(\tau_{1}, \tau_{2}\right)$ is called a thin-thick decomposition of $\tau$ if $\tau_{1}$ is a thin time, $\tau_{2}$ is a thick time, and

$$
\tau=\tau_{1} \wedge \tau_{2} \quad \tau_{1} \vee \tau_{2}=\infty
$$

Theorem 2.5. Any random time $\tau$ has a thin-thick decomposition $\left(\tau_{1}, \tau_{2}\right)$ which is a.s. unique.

Proof. Let us define $\tau_{1}$ and $\tau_{2}$ as $\tau_{1}:=\tau_{\left\{\Delta A_{\tau}^{o}>0\right\}}$ and $\tau_{2}:=\tau_{\left\{\Delta A_{\tau}^{o}=0\right\}}$, where $\tau_{C}$ is the restriction of the random time $\tau$ to the set $C$, defined as $\tau_{C}=\tau \mathbb{1}_{C}+\infty \mathbb{1}_{C^{c}}$. Properties of dual optional projection ensure that $\tau_{1}$ and $\tau_{2}$ satisfy the required conditions. More precisely, the time $\tau_{1}$ is a thin time since

$$
\llbracket \tau_{1} \rrbracket=\llbracket \tau \rrbracket \cap\left\{\Delta A^{o}>0\right\}=\llbracket \tau \rrbracket \cap \bigcup_{n} \llbracket T_{n} \rrbracket \subset \bigcup_{n} \llbracket T_{n} \rrbracket,
$$

where the sequence $\left(T_{n}\right)_{n}$ exhausts the jumps of the càdlàg increasing process $A^{o}$, i.e., $\left\{\Delta A^{o}>0\right\}=\bigcup_{n} \llbracket T_{n} \rrbracket$ and the time $\tau_{2}$ is a thick time since, for any $\mathbb{F}$-stopping time $T$,

$$
\begin{aligned}
\mathbb{P}\left(\tau_{2}=T<\infty\right) & =\mathbb{E}\left[\mathbb{1}_{\{\tau=T\} \cap\left\{\Delta A_{\tau}^{o}=0\right\}} \mathbb{1}_{(T<\infty)}\right] \\
& =\mathbb{E}\left[\int_{0}^{\infty} \mathbb{1}_{\{u=T\} \cap\left\{\Delta A_{u}^{o}=0\right\}} d A_{u}^{o}\right]=0 .
\end{aligned}
$$

In the following proposition we study the condition $A^{o}=A^{p}$. We have seen already that, if either $\tau$ avoids $\mathbb{F}$ stopping times or all $\mathbb{F}$-martingales are continuous, then this condition holds.

Proposition 2.6. The condition $A^{o}=A^{p}$ holds if and only if the random time $\tau$ satisfies that:

(a) $\mathbb{P}(\tau=T<\infty)=0$ for any $\mathbb{F}$-totally inaccessible stopping time $T$, and

(b) $\mathbb{P}\left(\tau=S<\infty \mid \mathcal{F}_{S}\right)=\mathbb{P}\left(\tau=S<\infty \mid \mathcal{F}_{S-}\right)$ for any $\mathbb{F}$-predictable stopping time $S$.

Proof. By Proposition C.2 and Theorem 2.2 it is enough to assume that $\tau$ is a thin time. We choose an exhausting sequence $\left(S_{n}\right)_{n \geq 1}$ so that it only contains totally inaccessible or predictable stopping times. Then, by Proposition C.1 (b) and by the fact that $A^{p}=\left(A^{o}\right)^{p}$, we conclude that $A^{o}=A^{p}$ is equivalent to $z_{S_{n}}^{n} \mathbb{1}_{\llbracket S_{n}, \infty \llbracket}=\left(z_{S_{n}}^{n} \mathbb{1}_{\llbracket S_{n}, \infty \llbracket}\right)^{p}$ for each $n \geq 1$. If $S_{n}$ is totally inaccessible then the latter condition is equivalent to $z_{S_{n}}^{n} \mathbb{1}_{\left\{S_{n}<\infty\right\}}=0$ which is the condition (1). If $S_{n}$ is predictable then $\left(z_{S_{n}}^{n} \mathbb{1}_{\llbracket S_{n}, \infty \llbracket}\right)^{p}={ }^{p} z_{S_{n}}^{n} \mathbb{1}_{\llbracket S_{n}, \infty \llbracket}$ which boils down to the condition (2).

Remark 2.7. The condition (b) from Proposition 2.6 is always satisfied in quasi-left continuous filtrations, since then $\mathcal{F}_{S}=\mathcal{F}_{S-}$ for $\mathbb{F}$-predictable stopping time $S$. Therefore, if $\mathbb{F}$ is the natural filtration of a Poisson process with jump times $\left(T_{n}\right)_{n \geq 1}$, which is quasi-left continuous, the condition $A^{o}=A^{p}$ holds if and only if $\mathbb{P}\left(\tau=T_{n}<\infty\right)=0$ for any $n$, since any $\mathbb{F}$-totally inaccessible stopping time $T$ satisfies $\llbracket T \rrbracket \subset \bigcup_{n} \llbracket T_{n} \rrbracket$. One can find such an example in [1, Proposition 4].

Remark 2.8. (a) Since $\tau_{1}$ is an $\mathbb{F}^{\tau}$-stopping time, it can be decomposed into $\mathbb{F}^{\tau}$-accessible and $\mathbb{F}^{\tau}$-totally inaccessible parts. Thus, we can consider the decomposition of $\tau$ into three parts as:

$$
\tau_{1}^{i}=\tau_{\left\{\Delta A_{\tau}^{o}>0, \Delta A_{\tau}^{p}=0\right\}}, \quad \tau_{1}^{a}=\tau_{\left\{\Delta A_{\tau}^{o}>0, \Delta A_{\tau}^{p}>0\right\}} \quad \text { and } \quad \tau_{2}=\tau_{\left\{\Delta A_{\tau}^{o}=0\right\}} .
$$

Since $\tau_{2}$ is $\mathbb{F}^{\tau}$-totally inaccessible, it follows that $\tau_{1}^{i} \wedge \tau_{2}$ is the $\mathbb{F}^{\tau}$-totally inaccessible part and $\tau_{1}^{a}$ is the $\mathbb{F}^{\tau}$-accessible part of the $\mathbb{F}^{\tau}$-stopping time $\tau$. Results of a similar type can 
be found in [6] and [17, p.65]. We note that $\tau$ is an $\mathbb{F}^{\tau}$-predictable stopping time if and only if $\tau$ is equal to an $\mathbb{F}$-predictable stopping time on $\{\tau>0\}$.

(b) Assume that the $\mathbb{F}^{\tau}$-accessible stopping time $\tau_{1}^{a}$ is not equal to an $\mathbb{F}$-stopping time on $\{\tau>0\}$. Then, the filtration $\mathbb{F}^{\tau}$ is not quasi-left continuous. This provides a systemic way to construct examples of non quasi-left continuous filtrations.

\section{Link between thin times and honest times}

In this section, we restrict our attention to a special class of random times, namely to honest times. We recall the definition below (see [17, p. 73]) and some alternative characterizations in Appendix B. Honest times are a well- studied class of time for which, in particular, the hypothesis $\left(\mathcal{H}^{\prime}\right)$ holds.

Definition 3.1. A random time $\tau$ is an $\mathbb{F}$-honest time if for every $t>0$ there exists an $\mathcal{F}_{t}$-measurable random variable $\tau_{t}$ such that $\tau=\tau_{t}$ on $\{\tau<t\}$.

Remark 3.2. By eventually taking $\tau_{t} \wedge t$, it is always possible to choose $\tau_{t}$ such that $\tau_{t} \leq t$ in Definition 3.1.

\subsection{Fundamental properties}

We start with providing a characterisation and properties of (thin) honest times.

Theorem 3.3. (a) Let $\left(\tau_{1}, \tau_{2}\right)$ be the thin-thick decomposition of $\tau$. Then, $\tau$ is honest if and only if $\tau_{1}$ and $\tau_{2}$ are honest.

(b) A random time $\tau$ is a thick honest time if and only if $Z_{\tau}=1$ a.s. on $\{\tau<\infty\}$.

(c) Let $\tau$ be an honest time with thin-thick decomposition $\left(\tau_{1}, \tau_{2}\right)$. Then, $Z_{\tau}<1$ on $\left\{\tau=\tau_{1}<\infty\right\}$ and $Z_{\tau}=1$ on $\left\{\tau=\tau_{2}<\infty\right\}$.

Proof. (a) On the set $\{\tau<\infty\}, \tau$ is equal to $\gamma$, the end of the optional set $\Gamma$ (Theorem B.2). Then, as $\left\{\tau_{1}<\infty\right\} \subset\{\tau<\infty\}$, on the set $\left\{\tau_{1}<\infty\right\}$, one has $\tau_{1}=\gamma$, so $\tau_{1}$ is an honest time. Same argument for $\tau_{2}$.

(b) Assume that $\tau$ is a thick honest time. Then, the honest time property presented in Theorem B.2 (c) implies that $\widetilde{Z}_{\tau}=1$ and the thick time property implies, by Theorem 1.4 (b), the continuity of $A^{o}$. Therefore, the equality $\widetilde{Z}=Z+\Delta A^{o}$ leads to equality $Z_{\tau}=1$ a.s. on $\{\tau<\infty\}$.

Assume now that $Z_{\tau}=1$ on the set $\{\tau<\infty\}$. Then, on $\{\tau<\infty\}$ we have $1=Z_{\tau} \leq$ $\widetilde{Z}_{\tau} \leq 1$, so $\widetilde{Z}_{\tau}=1$ and $\tau$ is an honest time. Furthermore, as $\Delta A_{\tau}^{o}=\widetilde{Z}_{\tau}-Z_{\tau}=0$, for each F-stopping time $T$ we have

$$
\begin{aligned}
\mathbb{P}(\tau=T<\infty) & =\mathbb{E}\left[\mathbb{1}_{\{\tau=T\}} \mathbb{1}_{\left\{\Delta A_{\tau}^{o}=0\right\}} \mathbb{1}_{(T<\infty)}\right] \\
& =\mathbb{E}\left[\int_{0}^{\infty} \mathbb{1}_{\{u=T\}} \mathbb{1}_{\left\{\Delta A_{u}^{o}=0\right\}} d A_{u}^{o}\right]=0 .
\end{aligned}
$$

So $\tau$ is a thick time.

(c) Denoting by $Z^{i}, \widetilde{Z}^{i}$ the supermartingales associated with $\tau_{i}$ for $i=1,2$, from the honest time property of $\tau$ and Proposition C.2 (a), on the set $\{\tau<\infty\}$

$$
1=\widetilde{Z}_{\tau}=\widetilde{Z}_{\tau}^{1}+\widetilde{Z}_{\tau}^{2}-1
$$

On the set $\left\{\tau=\tau_{1}<\infty\right\}$,

$$
Z_{\tau}=Z_{\tau_{1}}^{1}+Z_{\tau_{1}}^{2}-1 \leq Z_{\tau_{1}}^{2}<1
$$

where the last inequality is due to Proposition C.1 (a). On the set $\left\{\tau=\tau_{2}<\infty\right\}$, we have

$$
1=\widetilde{Z}_{\tau_{2}}^{1}+\widetilde{Z}_{\tau_{2}}^{2}-1=\widetilde{Z}_{\tau_{2}}^{1}
$$


where the second equality comes from (c) in Theorem B.2. Now let us compute $Z_{\tau_{2}}^{1}$

$$
Z_{\tau_{2}}^{1}=\widetilde{Z}_{\tau_{2}}^{1}-\Delta A_{\tau_{2}}^{1, o}=\widetilde{Z}_{\tau_{2}}^{1}=1,
$$

where we have used that $\left\{\Delta A^{1, o}>0\right\}=\bigcup_{n=1}^{\infty} \llbracket T_{n} \rrbracket$ with $\left(T_{n}\right)_{n \geq 0}$ being an exhausting sequence of $\tau_{1}$, and the thick time property of $\tau_{2}$, i.e., $\mathbb{P}\left(\tau_{2}=T_{n}<\infty\right)=0$. Finally, on $\left\{\tau=\tau_{2}<\infty\right\}$

$$
Z_{\tau}=Z_{\tau_{2}}^{1}+Z_{\tau_{2}}^{2}-1=1
$$

Remark 3.4. We would like to remark that the condition $Z_{\tau}<1$ for an honest time $\tau-$ which, by Theorem 3.3 (c), is equivalent to the condition that $\tau$ is a thin honest time - is an essential assumption in [2] for the study of arbitrages after honest times.

\subsection{Jumping filtration}

In this subsection we develop the relationship between jumping filtration and thin honest times. Let us first recall the definition of a jumping filtration studied in Jacod and Skorokhod [14].

Definition 3.5. A filtration $\mathbb{F}$ is called a jumping filtration if there exists a localizing sequence $\left(\theta_{n}\right)_{n \geq 0}$, i.e., a sequence of stopping times increasing a.s. to $\infty$, with $\theta_{0}=0$ and such that, for all $n$ and $t>0$, the $\sigma$-fields $\mathcal{F}_{t}$ and $\mathcal{F}_{\theta_{n}}$ coincide up to null sets on $\left\{\theta_{n} \leq t<\theta_{n+1}\right\}$.

The sequence $\left(\theta_{n}\right)_{n}$ is then called a jumping sequence.

We investigate relationship between jumping filtration and honest times. We show that there does not exist thick honest time in a jumping filtration and that there exists a thick honest time in a filtration which admits a non-constant continuous martingale (in particular such a filtration is not a jumping filtration).

Theorem 3.6. The following assertions hold.

(a) If $\mathbb{F}$ is a jumping filtration, then all $\mathbb{F}$-honest times are thin.

(b) If all $\mathbb{F}$-honest times are thin, then all $\mathbb{F}$-local martingales are purely discontinuous.

Proof. (a) Let $\tau$ be an honest time. Then, take the same process $\alpha$ as in the proof of Proposition B.1, i.e., $\alpha$ is an increasing, càdlàg, adapted process such that $\alpha_{t}=\tau$ on $\{\tau \leq t\}$ and $\tau=\sup \left\{t: \alpha_{t}=t\right\}$. Let us define the partition $\left(C_{n}\right)_{n=0}^{\infty}$ such that

$$
C_{n}=\left\{\theta_{n-1} \leq \tau<\theta_{n}\right\}
$$

for $n \geq 1$ and $C_{0}=\{\tau=\infty\}$ with $\left(\theta_{n}\right)_{n \geq 0}$ being a jumping sequence for the jumping filtration $\mathbb{F}$. On each $C_{n}$ with $n \geq 1$, we have

$$
\tau=T_{n}:=\inf \left\{t \geq \theta_{n-1}: t=\alpha_{\theta_{n}-}\right\} .
$$

From the jumping filtration property, we know that $\alpha_{\theta_{n}-}$ is $\mathcal{F}_{\theta_{n-1}}$-measurable, so each $T_{n}$ is a stopping time and $\llbracket \tau \rrbracket \subset \bigcup_{n=1}^{\infty} \llbracket T_{n} \rrbracket$ which shows that the honest time $\tau$ is a thin time.

(b) By [14, Theorem 1], $\mathbb{F}$ is a jumping filtration if and only if all $\mathbb{F}$-martingales has finite variation. Then, using this equivalence, the proof by contradiction is based on [26, Exercise (1.26) p.235]. Assume that $M$ is a non-constant continuous $\mathbb{F}$-local martingale with $M_{0}=0$. Define the $\mathbb{F}$-stopping time $S_{1}=\inf \left\{t>0:\langle M\rangle_{t}=1\right\}$. Then, define the F-honest time 
Thin times and random times' decomposition

$$
\tau:=\sup \left\{t \leq S_{1}: M_{t}=0\right\} .
$$

Since $M$ is continuous, $\tau$ is not equal to infinity with strictly positive probability. We now show that $\tau$ is an $\mathbb{F}$-thick honest time. Let us denote $\mathcal{Z}(\omega):=\left\{t: M_{t}(\omega)=0\right\}$. The set $\mathcal{Z}(\omega)$ is closed and $\mathcal{Z}^{c}(\omega)$ is the union of countably many open intervals. We call $G(\omega)$ the set of left ends of these open intervals. In what follows we show that for any $\mathbb{F}$-stopping time $T$, we have $\mathbb{P}(T \in G)=0$. Define the $\mathbb{F}$-stopping time $D_{T}:=\inf \left\{t>T: M_{t}=0\right\}$ and note that $\{T \in G\}=\left\{M_{T}=0\right\} \cap\left\{T<D_{T}\right\} \in \mathcal{F}_{T}$. Assume $\mathbb{P}(T \in G)=p>0$. Then the process

$$
Y_{t}=\mathbb{1}_{\{T \in G\}}\left|M_{T+t}\right| \mathbb{1}_{\left\{0 \leq t \leq D_{T}-T\right\}}
$$

is an $\left(\mathcal{F}_{T+t}\right)_{t \geq 0}$-martingale. Indeed, for $s \leq t$, we have

$$
\begin{aligned}
\mathbb{E}\left(Y_{t} \mid \mathcal{F}_{T+s}\right)=\mathbb{1}_{\{T \in G\}} \operatorname{sgn}\left(M_{T+t}\right) \mathbb{E}\left(M_{T+t} \mathbb{1}_{\left\{t \leq D_{T}-T\right\}} \mid \mathcal{F}_{T+s}\right) \\
=\mathbb{1}_{\{T \in G\}} \operatorname{sgn}\left(M_{T+t}\right) \\
\quad \quad\left[M_{T+s} \mathbb{1}_{\left\{s \leq D_{T}-T\right\}}-\mathbb{E}\left(\mathbb{1}_{\left\{s \leq D_{T}-T\right\}} \mathbb{1}_{\left\{t>D_{T}-T\right\}} \mathbb{E}\left(M_{T+t} \mid \mathcal{F}_{D_{T}}\right) \mid \mathcal{F}_{T+s}\right)\right] \\
=Y_{s}-\mathbb{1}_{\{T \in G\}} \operatorname{sgn}\left(M_{T+t}\right) \mathbb{E}\left(\mathbb{1}_{\left\{s \leq D_{T}-T\right\}} \mathbb{1}_{\left\{t>D_{T}-T\right\}} M_{D_{T}} \mid \mathcal{F}_{T+s}\right) \\
=Y_{s}
\end{aligned}
$$

where we have used the martingale property of $M$ and $M_{D_{T}}=0$. Moreover $Y_{0}=0$ and there exists $\varepsilon>0$ such that

$$
\mathbb{P}\left(M_{T}=0, D_{T}-T>\varepsilon\right) \geq \frac{p}{2}>0
$$

Since $Y_{\varepsilon}=\mathbb{1}_{\left\{M_{T}=0\right\}} \mathbb{1}_{\left\{D_{T}-T \geq \varepsilon\right\}}\left|M_{T+\varepsilon}\right| \geq 0$ and $\mathbb{P}\left(Y_{\varepsilon}>0\right)>0$, we have $\mathbb{E}\left(Y_{\varepsilon}\right)>0=$ $Y_{0}$. So, $\mathbb{P}(T \in G)=0$. Finally, as $\tau \in G$ a.s. we conclude that $\tau$ is a thick honest time.

Finally we give two examples of thick honest times originating from purely discontinuous semimartingales of infinite variation. In particular, these examples show that a reverse implication in Theorem 3.6(b) does not hold. In the first Example 3.7, we study the case of Azéma's martingale (see [25, IV.8 p.232-237]). In the second Example 3.8, we recall Example 2.1 from [19] on Maximum of downwards drifting spectrally negative Lévy processes with paths of infinite variation.

Example 3.7. Let $B$ be a Brownian motion and $\mathbb{F}$ its natural filtration. Define the process

$$
g_{t}:=\sup \left\{s \leq t: B_{s}=0\right\}
$$

The process

$$
\mu_{t}:=\operatorname{sgn}\left(B_{t}\right) \sqrt{t-g_{t}}
$$

is a martingale with respect to the filtration $\mathbb{G}:=\left(\mathcal{F}_{g_{t}+}\right)_{t \geq 0}$ and is called the Azéma martingale. Then, the random time

$$
\tau:=\sup \left\{t \leq 1: \mu_{t}=0\right\}
$$

is clearly a $\mathbb{G}$-honest time. Note that $\tau=\tau^{B}:=\sup \left\{t \leq 1: B_{t}=0\right\}$ and $\tau^{B}$ is an $\mathbb{F}$-thick honest time since it has continuous $\mathbb{F}$-dual optional projection (see in [21, Table $1 \alpha 1$ ), p.32]). Thus, since $\mathbb{G} \subset \mathbb{F}, \tau$ is a $\mathbb{G}$-thick honest time.

Example 3.8. Let $X$ be a Lévy process with characteristic triplet $\left(\alpha, \sigma^{2}=0, \nu\right)$ satisfying $\nu((0, \infty))=0, \alpha+\int_{-\infty}^{-1} x \nu(d x)<0$ and $\int_{-1}^{0}|x| \nu(d x)=\infty$. Then, $\rho=\sup \left\{t: X_{t-}=X_{t-}^{*}\right\}$ with $X_{t}^{*}=\sup _{s \leq t} X_{s}$ is a thick honest time as shown in [19, Section 2.1]. 


\section{Martingale and semimartingale stability for thin times}

\subsection{The hypothesis $\left(\mathcal{H}^{\prime}\right)$ for thin times}

One of the vital questions in the enlargement of filtration theory is whether all semimartingales in the reference filtration remain semimartingales in an enlarged filtration, i.e., whether the hypothesis $\left(\mathcal{H}^{\prime}\right)$ holds. In progressive enlargement setting there are only few classes of random times with this property, i.e., honest times and random times satisfying Jacod's absolutely continuous condition. In this section we prove the hypothesis $\left(\mathcal{H}^{\prime}\right)$ for the new class of random times, i.e., thin times.

Theorem 4.1. Let $\tau$ be a thin time. Then the hypothesis $\left(\mathcal{H}^{\prime}\right)$ is satisfied for $\left(\mathbb{F}, \mathbb{F}^{\tau}\right)$. Moreover, each $\mathbb{F}$-local martingale $X$ has the following $\mathbb{F}^{\tau}$-semimartingale canonical decomposition

$$
X_{t}=\widehat{X}_{t}+\int_{0}^{t \wedge \tau} \frac{1}{Z_{s-}} d\langle X, m\rangle_{s}+\sum_{n=1}^{\infty} \mathbb{1}_{C_{n}} \int_{0}^{t} \mathbb{1}_{\left\{s>T_{n}\right\}} \frac{1}{z_{s-}^{n}} d\left\langle X, z^{n}\right\rangle_{s}
$$

where $\widehat{X}$ is an $\mathbb{F}^{\tau}$-local martingale and $\left(z^{n}\right)_{n \geq 0}$ is a martingale family of the thin time $\tau$, and the predictable brackets are computed in $\mathbb{F}$.

Proof. Let $\mathbb{F}^{\mathcal{C}}$ denote the initial enlargement of the filtration $\mathbb{F}$ with the atomic $\sigma$-field $\mathcal{C}:=\sigma\left(C_{n}, n \geq 0\right)$ generated by a partition $\left(C_{n}, n \geq 0\right)$ of a thin time $\tau$, i.e.,

$$
\mathcal{F}_{t}^{\mathcal{C}}:=\bigcap_{s>t} \mathcal{F}_{s} \vee \sigma\left(C_{n}, n \geq 0\right)
$$

For this case of enlargement, Jacod's result (see [17, Theorem 3,2] and [22]) implies that the hypothesis $\left(\mathcal{H}^{\prime}\right)$ holds for $\left(\mathbb{F}, \mathbb{F}^{\mathcal{C}}\right)$. Since clearly $\mathbb{F} \subset \mathbb{F}^{\tau} \subset \mathbb{F}^{\mathcal{C}}$, Stricker's Theorem [25, Theorem 4, Chapter II] implies that the hypothesis $\left(\mathcal{H}^{\prime}\right)$ holds for $\left(\mathbb{F}, \mathbb{F}^{\tau}\right)$. To prove the decomposition result, let $H$ be an $\mathbb{F}^{\tau}$-predictable bounded process. Then, [17, Lemma $(4,4)]$ implies that

$$
H_{t}=\mathbb{1}_{\{t \leq \tau\}} J_{t}+\mathbb{1}_{\{\tau<t\}} K_{t}(\tau), \quad t \geq 0,
$$

where $J$ is an $\mathbb{F}$-predictable bounded process and $K: \mathbb{R}_{+} \times \Omega \times \mathbb{R}_{+} \rightarrow \mathbb{R}$ is $\mathcal{P} \otimes \mathcal{B}\left(\mathbb{R}_{+}\right)$measurable and bounded ( $\mathcal{P}$ denotes the $\mathbb{F}$-predictable $\sigma$-field). Since $\tau$ is a thin time, we can rewrite the process $H$ as

$$
H_{t}=J_{t} \mathbb{1}_{\{t \leq \tau\}}+\sum_{n=1}^{\infty} \mathbb{1}_{\left\{T_{n}<t\right\}} K_{t}\left(T_{n}\right) \mathbb{1}_{C_{n}} .
$$

Note that, since $\{t \leq \tau\} \subset\left\{Z_{t-}>0\right\}, J$ can be chosen to satisfy $J_{t}=J_{t} \mathbb{1}_{\left\{Z_{t-}>0\right\}}$ and, since $C_{n} \subset\left\{z_{t-}^{n}>0\right\}$, each process $K_{t}^{n}:=\mathbb{1}_{\left\{T_{n}<t\right\}} K_{t}\left(T_{n}\right)$ being $\mathbb{F}$-predictable and bounded, $K^{n}$ can be chosen to satisfy $K_{t}^{n}=K_{t}^{n} \mathbb{1}_{\left\{z_{t-}^{n}>0\right\}}$.

We denote by $H^{1}(\mathbb{F})$ the space of $\mathbb{F}$-local martingales $N$ s.t. $\mathbb{E}\left([N]_{\infty}^{1 / 2}\right)<\infty$. Let $N$ be an $H^{1}(\mathbb{F})$-martingale. Then the stochastic integrals $J \cdot N$ and $K^{n} \cdot N$ are well defined and each of them is an $H^{1}(\mathbb{F})$-martingale. For each $n \geq 0$ and for each bounded $\mathbb{F}$-martingale $N$, by integration by parts, we have that

$$
\mathbb{E}\left[\mathbb{1}_{C_{n}} N_{\infty}\right]=\mathbb{E}\left[\left[z^{n}, N\right]_{\infty}\right]=\mathbb{E}\left[\left\langle z^{n}, N\right\rangle_{\infty}\right] .
$$

Since $N \rightarrow \mathbb{E}\left[\mathbb{1}_{C_{n}} N_{\infty}\right]$ is a linear form, the duality $\left(H^{1}, B M O\right)$ implies that (4.3) holds for any $H^{1}(\mathbb{F})$-martingale $N$. Similarly, since $m$, given in (1.1), is a $B M O(\mathbb{F})$-martingale, for any $H^{1}(\mathbb{F})$-martingale $N$, the process $\langle N, m\rangle$ exists and we have

$$
\mathbb{E}\left[N_{\tau}\right]=\mathbb{E}\left[[N, m]_{\infty}\right]=\mathbb{E}\left[\langle N, m\rangle_{\infty}\right] .
$$


Therefore

$$
\begin{aligned}
\mathbb{E}\left[\int_{0}^{\infty} H_{s} d N_{s}\right] & =\mathbb{E}\left[\int_{0}^{\tau} J_{s} d N_{s}\right]+\sum_{n=1}^{\infty} \mathbb{E}\left[\mathbb{1}_{C_{n}} \int_{0}^{\infty} K_{s}^{n} d N_{s}\right] \\
& =\mathbb{E}\left[\int_{0}^{\infty} J_{s} d\langle m, N\rangle_{s}\right]+\sum_{n=1}^{\infty} \mathbb{E}\left[\int_{0}^{\infty} K_{s}^{n} d\left\langle z^{n}, N\right\rangle_{s}\right]
\end{aligned}
$$

Then, since for any predictable finite variation process $V, \mathbb{E}\left[\int_{0}^{\infty} h_{s} d V_{s}\right]=\mathbb{E}\left[\int_{0}^{\infty}{ }^{p} h_{s} d V_{s}\right]$, and $Z_{-}={ }^{p}\left(1-A_{-}\right)$, we deduce, taking care on the specific choice of $J$ and $K$,

$$
\begin{aligned}
\mathbb{E}\left[\int_{0}^{\infty} H_{s} d N_{s}\right]=\mathbb{E} & {\left[\int_{0}^{\infty} \frac{Z_{s-}}{Z_{s-}} \mathbb{1}_{\left\{Z_{s-}>0\right\}} J_{s} d\langle m, N\rangle_{s}\right] } \\
& +\sum_{n=1}^{\infty} \mathbb{E}\left[\int_{0}^{\infty} \frac{z_{s-}^{n}}{z_{s-}^{n}} \mathbb{1}_{\left\{z_{s-}^{n}>0\right\}} K_{s}^{n} d\left\langle z^{n}, N\right\rangle_{s}\right] \\
= & \mathbb{E}\left[\int_{0}^{\tau} \frac{1}{Z_{s-}} J_{s} d\langle m, N\rangle_{s}\right]+\sum_{n=1}^{\infty} \mathbb{E}\left[\mathbb{1}_{C_{n}} \int_{0}^{\infty} \frac{1}{z_{s-}^{n}} K_{s}^{n} d\left\langle z^{n}, N\right\rangle_{s}\right] .
\end{aligned}
$$

The assertion of the theorem follows as, for any $s \leq t$ and $F \in \mathcal{F}_{s}^{\tau}$, the process $H=$ $\mathbb{1}_{(s, t]} \mathbb{1}_{F}$ is clearly $\mathbb{F}^{\tau}$-predictable. To end the proof, we recall that any local martingale is locally in $H^{1}$ (see [25, Theorem 51, Chapter IV]).

Remark 4.2. Lemma $(4,11)$ in [17], where the random time with countably many values is considered, is a special case of Theorem 4.1. It corresponds to the situation of thin random time whose graph is included in countable union of constant sections, i.e, $\llbracket \tau \rrbracket \subset \bigcup_{n} \llbracket t_{n} \rrbracket$ with $\llbracket t_{n} \rrbracket=\left\{\left(\omega, t_{n}\right): \omega \in \Omega\right\}$ and $t_{n} \in \mathbb{R}^{+}$.

We end this section with a second proof of Theorem 4.1. It is based on Lemma 4.3 which establishes a link between processes in $\mathbb{F}^{\tau}$ and $\mathbb{F}^{\mathcal{C}}$.

Lemma 4.3. Let $\tau$ be a thin time and $Y$ be a process such that $Y=\mathbb{1}_{\rrbracket \tau, \infty \llbracket} \cdot Y$. Then:

(a) The process $Y$ is an $\mathbb{F}^{\mathcal{C}}$-(super, sub)martingale if and only if the process $Y$ is an $\mathbb{F}^{\tau}$-(super, sub)martingale.

(b) Let $\vartheta$ be an $\mathbb{F}^{\mathcal{C}}$-stopping time. Then $\vartheta \vee \tau$ is an $\mathbb{F}^{\tau}$-stopping time.

(c) The process $Y$ is an $\mathbb{F}^{\mathcal{C}}$-local martingale if and only if the process $Y$ is an $\mathbb{F}^{\tau}$-local martingale.

Proof. (a) Note that the filtrations $\mathbb{F}^{\tau}$ and $\mathbb{F}^{\mathcal{C}}$ are equal after $\tau$, i.e., for each $t$ and for each set $G \in \mathcal{F}_{t}^{\mathcal{C}}$, there exists a set $F \in \mathcal{F}_{t}^{\tau}$ such that

$$
\{\tau \leq t\} \cap G=\{\tau \leq t\} \cap F .
$$

To show (4.4), by monotone class theorem, it is enough to consider $G=C_{n}$ and to take $F=C_{n} \cap\{\tau \leq t\}$ which belongs to $\mathcal{F}_{t}^{\tau}$ as $C_{n} \in \mathcal{F}_{\tau}^{\tau}$ by [11, Corollary 3.5]. That implies that the process $\mathbb{1}_{\rrbracket \tau, \infty \mathbb{I}} \cdot Y$ is $\mathbb{F}^{\tau}$-adapted if and only if it is $\mathbb{F}^{\mathcal{C}}$-adapted. The equivalence of (super-, sub-) martingale property comes from (4.4).

(b) For each $t$ we have $\{\vartheta \vee \tau \leq t\}=\{\vartheta \leq t\} \cap\{\tau \leq t\} \in \mathcal{F}_{t}^{\tau}$ by (4.4).

(c) We combine the two previous points.

Second proof of Theorem 4.1. Let $X$ be a bounded $\mathbb{F}$-martingale. Then, it is enough to show that $\mathbb{1}_{\llbracket 0, \tau \rrbracket} \cdot X$ and $\mathbb{1}_{\rrbracket \tau, \infty \llbracket} \cdot X$ are two $\mathbb{F}^{\tau}$-semimartingales with appropriate 
decompositions. By [17, Proposition $(4,16)] \mathbb{1}_{\llbracket 0, \tau \rrbracket} \cdot X=X_{\cdot \wedge \tau}$ is an $\mathbb{F}^{\tau}$-semimartingale with the decomposition

$$
X_{t \wedge \tau}=\widehat{X}_{t}^{1}+\int_{0}^{t \wedge \tau} \frac{1}{Z_{s-}} d\langle X, m\rangle_{s}
$$

where $\widehat{X}^{1}$ is an $\mathbb{F}^{\tau}$-local martingale. By Lemma 4.3 and Jacod's result (see [17, Theorem $3,2]$ and [22]), it follows that $\mathbb{1}_{\rrbracket \tau, \infty} \cdot X$ is an $\mathbb{F}^{\tau}$-semimartingale with decomposition

$$
X_{t}-X_{t \wedge \tau}=\widehat{X}_{t}^{2}+\sum_{n=1}^{\infty} \mathbb{1}_{C_{n}} \int_{0}^{t} \mathbb{1}_{\left\{s>T_{n}\right\}} \frac{1}{z_{s-}^{n}} d\left\langle X, z^{n}\right\rangle_{s},
$$

where $\widehat{X}^{2}$ is an $\mathbb{F}^{\tau}$-local martingale. This completes the proof.

\subsection{Immersion for thin times}

Immersion, also called the hypothesis $(\mathcal{H})$ is a more restrictive hypothesis for enlargement of filtration than the hypothesis $\left(\mathcal{H}^{\prime}\right)$. Given $\mathbb{F} \subset \mathbb{G}$, we say that $\mathbb{F}$ is immersed in $\mathbb{G}$ if any $\mathbb{F}$-martingale is a $\mathbb{G}$-martingale. The equivalent condition to immersion, established in Theorem 3 in [5], says that for each $t \geq 0$ and $G \in L^{1}\left(\mathcal{G}_{t}\right)$ it holds that $\mathbb{E}\left[G \mid \mathcal{F}_{t}\right]=\mathbb{E}\left[G \mid \mathcal{F}_{\infty}\right]$. Immersion does not hold for each thin time. However, in the next proposition, an equivalent condition to immersion is given. In particular, it implies that there exist thin times for which immersion holds and which are not stopping times.

Proposition 4.4. Let $\tau$ be a thin time with exhausting sequence $\left(T_{n}\right)_{n \geq 0}$, partition $\left(C_{n}\right)_{n \geq 0}$ and martingale family $\left(z^{n}\right)_{n \geq 0}$. Then, $\mathbb{F}$ is immersed in $\mathbb{F}^{\tau}$ if and only if one of the following conditions hold:

(a) $z_{\infty}^{n}=z_{T_{n}}^{n}$ for each $n \geq 1$,

(b) $z_{t}^{n}=z_{T_{n} \wedge t}^{n}$ for each $t \geq 0$ for each $n \geq 1$,

(c) for each $n \geq 1, C_{n}$ is independent of $\mathcal{F}_{\infty}$ conditionally w.r.t. $\mathcal{F}_{T_{n}}$.

Proof. By Theorem 3 in [5], Lemma 1.5 (b) and monotone class theorem, $\mathbb{F}$ is immersed in $\mathbb{F}^{\tau}$ if and only if $\mathbb{P}\left(C_{n} \cap\left\{T_{n} \leq t\right\} \mid \mathcal{F}_{t}\right)=\mathbb{P}\left(C_{n} \cap\left\{T_{n} \leq t\right\} \mid \mathcal{F}_{\infty}\right)$ for each $n \geq 1$. The last condition is precisely $z_{t}^{n} \mathbb{1}_{\left\{T_{n} \leq t\right\}}=z_{\infty}^{n} \mathbb{1}_{\left\{T_{n} \leq t\right\}}$ for each $n \geq 1$, which is the condition (b). Since $z^{n}$ are martingales, we conclude that immersion is equivalent to $z_{T_{n}}^{n}=z_{\infty}^{n}$ stated in the condition (a). Since $z_{T_{n}}^{n}=z_{\infty}^{n}$ can be rewritten as $\mathbb{P}\left(C_{n} \mid \mathcal{F}_{T_{n}}\right)=\mathbb{P}\left(C_{n} \mid \mathcal{F}_{\infty}\right)$, we conclude that immersion is satisfied if and only if, for each $n \geq 1, C_{n}$ is independent of $\mathcal{F}_{\infty}$ conditionally w.r.t. $\mathcal{F}_{T_{n}}$.

Remark 4.5. Immersion property for a random time $\tau$ implies in particular that $\tau$ is a pseudo-stopping time. Recall that a random time $\tau$ is a pseudo-stopping time if for any bounded $\mathbb{F}$-martingale $X$ it holds that $\mathbb{E}\left[X_{\tau}\right]=\mathbb{E}\left[X_{0}\right]$, or equivalently as established in [24], if $m \equiv 1$.

Let $\tau$ be a thin time. Then, by Proposition C.1, $\tau$ is a pseudo-stopping time if and only if $\sum_{n=0}^{\infty} z_{t \wedge T_{n}}^{n}=1$ for any $t \geq 0$. Clearly, immersion implies the last condition as, by Proposition 4.4 (b) and since $T_{0}=\infty$,

$$
\sum_{n=0}^{\infty} z_{t \wedge T_{n}}^{n}=z_{t}^{0}+\sum_{n=1}^{\infty} z_{t \wedge T_{n}}^{n}=\sum_{n=0}^{\infty} z_{t}^{n}=1
$$

Reverse implication does not hold. 


\section{Martingale and semimartingale stability for a general random time}

\subsection{The hypothesis $\left(\mathcal{H}^{\prime}\right)$ for a random time}

We study here the hypothesis $\left(\mathcal{H}^{\prime}\right)$ in the progressive enlargement of filtration in connection to the thin-thick decomposition of the random time. Let $\left(\tau_{1}, \tau_{2}\right)$ be the thin-thick decomposition of a random time $\tau$. We define three enlarged filtrations $\mathbb{F}^{\tau_{1}}:=\left(\mathcal{F}_{t}^{\tau_{1}}\right)_{t \geq 0}, \mathbb{F}^{\tau_{2}}:=\left(\mathcal{F}_{t}^{\tau_{2}}\right)_{t \geq 0}$ and $\mathbb{F}^{\tau_{1}, \tau_{2}}:=\left(\mathcal{F}_{t}^{\tau_{1}, \tau_{2}}\right)_{t \geq 0}$ as

$$
\begin{aligned}
\mathcal{F}_{t}^{\tau_{i}}: & =\bigcap_{s>t} \mathcal{F}_{s} \vee \sigma\left(\tau_{i} \wedge s\right) \text { for } i=1,2 \\
\mathcal{F}_{t}^{\tau_{1}, \tau_{2}}: & :=\bigcap_{s>t} \mathcal{F}_{s} \vee \sigma\left(\tau_{1} \wedge s\right) \vee \sigma\left(\tau_{2} \wedge s\right) .
\end{aligned}
$$

Clearly, $\mathbb{F} \subset \mathbb{F}^{\tau_{i}} \subset \mathbb{F}^{\tau_{1}, \tau_{2}}=\left(\mathbb{F}^{\tau_{1}}\right)^{\tau_{2}}=\left(\mathbb{F}^{\tau_{2}}\right)^{\tau_{1}}$ for $i=1,2$.

Theorem 5.1. Let $\tau$ be a random time and $\left(\tau_{1}, \tau_{2}\right)$ its thin-thick decomposition. Then, $\mathbb{F}^{\tau}=\mathbb{F}^{\tau_{1}, \tau_{2}}$. Furthermore, the hypothesis $\left(\mathcal{H}^{\prime}\right)$ is satisfied for $\left(\mathbb{F}, \mathbb{F}^{\tau}\right)$ if and only if the hypothesis $\left(\mathcal{H}^{\prime}\right)$ is satisfied for $\left(\mathbb{F}, \mathbb{F}^{\tau_{2}}\right)$.

Proof. In a first step, we show that, for $i=1,2$ :

$$
\mathbb{F} \subset \mathbb{F}^{\tau_{i}} \subset \mathbb{F}^{\tau_{1}, \tau_{2}}=\mathbb{F}^{\tau}
$$

Let $A^{o}$ be the $\mathbb{F}$-dual optional projection of $\tau$. Note that

$$
\mathbb{1}_{\llbracket \tau_{1}, \infty \llbracket}=\mathbb{1}_{\llbracket \tau, \infty \llbracket} \mathbb{1}_{\left\{\Delta A_{\tau}^{o}>0\right\}} \quad \text { and } \quad \mathbb{1}_{\llbracket \tau_{2}, \infty \llbracket}=\mathbb{1}_{\llbracket \tau, \infty \llbracket} \mathbb{1}_{\left\{\Delta A_{\tau}^{o}=0\right\}},
$$

thus, since $\Delta A_{\tau}^{o} \in \mathcal{F}_{\tau}^{\tau}$, the processes $\mathbb{1}_{\llbracket \tau_{1}, \infty \llbracket}$ and $\mathbb{1}_{\llbracket \tau_{2}, \infty \llbracket}$ are $\mathbb{F}^{\tau}$-adapted which implies that $\mathbb{F}^{\tau_{1}, \tau_{2}} \subset \mathbb{F}^{\tau}$. On the other hand, we have

$$
\mathbb{1}_{\llbracket \tau_{1}, \infty \llbracket}+\mathbb{1}_{\llbracket \tau_{2}, \infty \llbracket}=\mathbb{1}_{\llbracket \tau, \infty \llbracket}
$$

which implies that $\mathbb{F}^{\tau_{1}, \tau_{2}} \supset \mathbb{F}^{\tau}$.

In a second step, note that if an $\mathbb{F}$-martingale is an $\mathbb{F}^{\tau}$-semimartingale, by Stricker's Theorem [25, Theorem 4, Chapter II, p. 53], it is as well an $\mathbb{F}^{\tau_{2}}$-semimartingale. Thus the necessary condition follows. Since $\tau_{1}$ is an $\mathbb{F}$-thin time, it is an $\mathbb{F}^{\tau_{2}}$-thin time and the equality $\mathbb{F}^{\tau_{1}, \tau_{2}}=\mathbb{F}^{\tau}$ and Theorem 4.1 imply that the hypothesis $\left(\mathcal{H}^{\prime}\right)$ is satisfied for $\left(\mathbb{F}^{\tau_{2}}, \mathbb{F}^{\tau}\right)$. Thus the sufficient condition follows.

In the following corollary, we examine the hypothesis $\left(\mathcal{H}^{\prime}\right)$ for the minimum of a thin time and a random time satisfying the hypothesis $\left(\mathcal{H}^{\prime}\right)$, namely an honest time or a time satisfying Jacod's absolute continuity condition (see [17, Chapter 5] and [15, 12] respectively).

Corollary 5.2. Let $\tau$ be a thin time and $\sigma$ be an honest time or a time satisfying Jacod's absolute continuity condition. Then, the hypothesis $\left(\mathcal{H}^{\prime}\right)$ is satisfied for $\left(\mathbb{F}, \mathbb{F}^{\tau \wedge \sigma}\right)$.

Proof. First we recall that, if $\sigma$ is honest then the hypothesis $\left(\mathcal{H}^{\prime}\right)$ is satisfied for $\left(\mathbb{F}, \mathbb{F}^{\sigma}\right)$ by [17, Theorem $(5,10)]$, and if $\sigma$ satisfies Jacod's absolute continuity condition then $\left(\mathcal{H}^{\prime}\right)$ is satisfied for $\left(\mathbb{F}, \mathbb{F}^{\sigma}\right)$ by [15, Theorem 3.1].

Let $\left(\sigma_{1}, \sigma_{2}\right)$ be a thin-thick decomposition of $\sigma$. Then, by Remark 1.3, $\tau \wedge \sigma_{1}$ is a thin time and $\left(\tau \wedge \sigma_{1}, \sigma_{2}\right)$ is a thin-thick decomposition of $\tau \wedge \sigma$. Then the statement of the corollary follows by applying twice Theorem 5.1 . 
Proposition 5.3. Let $\tau$ be a random time and $\left(\tau_{1}, \tau_{2}\right)$ its thin-thick decomposition. Let $\left(T_{n}\right)_{n \geq 0}$ be an $\mathbb{F}$-exhausting sequence, $\left(C_{n}\right)_{n \geq 0}$ an $\mathbb{F}$-partition and $\left(z^{n}\right)_{n \geq 0}$ an $\mathbb{F}$ martingale family of $\mathbb{F}$-thin time $\tau_{1}$. Assume that for an $\mathbb{F}$-local martingale $X$, there exists an $\mathbb{F}^{\tau_{2}}$-predictable finite variation process $\Gamma(X)$ such that $X=\widetilde{X}+\Gamma(X)$ where $\widetilde{X}$ is an $\mathbb{F}^{\tau_{2}}$-local martingale. Then,

$$
X_{t}=\widehat{X}_{t}+\Gamma(X)_{t}+\int_{0}^{t \wedge \tau} \frac{1}{Z_{s-}} d\langle\widetilde{X}, \widetilde{m}\rangle_{s}^{\mathbb{F}^{\tau_{2}}}+\sum_{n=1}^{\infty} \mathbb{1}_{C_{n}} \int_{0}^{t} \mathbb{1}_{\left\{s>T_{n}\right\}} \frac{1}{\widetilde{z}_{s-}^{n}} d\left\langle\widetilde{X}, \widetilde{z}^{n}\right\rangle_{s}^{\mathbb{F}^{\tau_{2}}}
$$

where $\widehat{X}$ is an $\mathbb{F}^{\tau}$-local martingale, $\widetilde{z}_{t}^{n}:=\mathbb{P}\left(C_{n} \mid \mathcal{F}_{t}^{\tau_{2}}\right)=\mathbb{1}_{\left\{t<\tau_{2}\right\}} \frac{z_{t}^{n}}{Z_{t}^{2}}$ and $\widetilde{m}_{t}=\sum_{n} \widetilde{z}_{t \wedge T_{n}}^{n}$.

Proof. The decomposition of $X$ as an $\mathbb{F}^{\tau}$-semimartingale follows by $\mathbb{F}^{\tau}=\mathbb{F}^{\tau_{1}, \tau_{2}}$ and Theorem 4.1 since $\tau_{1}$ is an $\mathbb{F}^{\tau_{2}}$-thin time. Lemma C. 3 and Proposition C.1 imply the forms of $\widetilde{z}^{n}$ and $\widetilde{m}$.

\subsection{Immersion for a random time}

Thin-thick decomposition finds application in studying immersion for a generic random time.

Proposition 5.4. $\mathbb{F}$ is immersed in $\mathbb{F}^{\tau}$ if and only if $\mathbb{F}$ is immersed in $\mathbb{F}^{\tau_{1}}$ and in $\mathbb{F}^{\tau_{2}}$. In that case, $\mathbb{F}^{\tau_{1}}$ and $\mathbb{F}^{\tau_{2}}$ are immersed in $\mathbb{F}^{\tau}$.

Proof. Since $\mathbb{F} \subset \mathbb{F}^{\tau_{i}} \subset \mathbb{F}^{\tau}$, it is clear that, if $\mathbb{F}$ is immersed in $\mathbb{F}^{\tau}$, then $\mathbb{F}$ is immersed in $\mathbb{F}^{\tau_{1}}$ and in $\mathbb{F}^{\tau_{2}}$.

Let $\mathbb{F}$ be immersed in $\mathbb{F}^{\tau_{1}}$ and in $\mathbb{F}^{\tau_{2}}$, i.e., $Z_{t}^{i}=\mathbb{P}\left(\tau_{i}>t \mid \mathcal{F}_{\infty}\right)$ for each $t \geq 0$, for $i=1,2$. Then, by Proposition C.2 (a),

$$
Z_{t}=Z_{t}^{1}+Z_{t}^{2}-1=\mathbb{P}\left(\tau_{1}>t \mid \mathcal{F}_{\infty}\right)+\mathbb{P}\left(\tau_{2}>t \mid \mathcal{F}_{\infty}\right)-1=\mathbb{P}\left(\tau>t \mid \mathcal{F}_{\infty}\right)
$$

and we conclude that $\mathbb{F}$ is immersed in $\mathbb{F}^{\tau}$.

It remains to prove the last assertion. Let $\mathbb{F}$ be immersed in $\mathbb{F}^{\tau}$. Then, using similar arguments as in the proof of Lemma C.3, we obtain:

$$
\mathbb{P}\left(\tau_{2}>t \mid \mathcal{F}_{\infty}^{\tau_{1}}\right)=\mathbb{1}_{\left\{\tau_{1} \leq t\right\}}+\mathbb{1}_{\left\{t<\tau_{1}\right\}} \frac{\mathbb{P}\left(\tau>t \mid \mathcal{F}_{\infty}\right)}{\mathbb{P}\left(\tau_{1}>t \mid \mathcal{F}_{\infty}\right)}
$$

and the assumed immersion yield to

$$
\mathbb{P}\left(\tau_{2}>t \mid \mathcal{F}_{\infty}^{\tau_{1}}\right)=\mathbb{1}_{\left\{\tau_{1} \leq t\right\}}+\mathbb{1}_{\left\{t<\tau_{1}\right\}} \frac{\mathbb{P}\left(\tau>t \mid \mathcal{F}_{t}\right)}{\mathbb{P}\left(\tau_{1}>t \mid \mathcal{F}_{t}\right)}=\mathbb{P}\left(\tau_{2}>t \mid \mathcal{F}_{t}^{\tau_{1}}\right) .
$$

Therefore, $\mathbb{F}^{\tau_{1}}$ is immersed in $\mathbb{F}^{\tau}$. The same proof is valid for $\tau_{2}$.

Remark 5.5. In [18], the authors introduce a random time $\tau=\vartheta \wedge \xi$ where $\xi$ avoids F-stopping times, and is constructed as $\xi=\inf \left\{t: \Lambda_{t}:=\int_{0}^{t} \lambda_{s} d s \geq \Theta\right\}$ where $\lambda$ is a positive $\mathbb{F}$-adapted process and $\Theta$ is an exponential random variable independent from $\mathbb{F}$, and $\vartheta$ is an $\mathbb{F}$-accessible stopping time. Therefore, $\xi$ is thick and $\vartheta$ is thin. The thin-thick decomposition $\tau=\tau_{1} \wedge \tau_{2}$ can be obtained as follows: $\tau_{2}=\vartheta \mathbb{1}_{\{\vartheta<\xi\}}+\infty \mathbb{1}_{\{\xi \leq \vartheta\}}$ and $\tau_{1}=\xi \mathbb{1}_{\{\xi<\vartheta\}}+\infty \mathbb{1}_{\{\vartheta \leq \xi\}}$.

The authors establish immersion property by checking $\mathbb{P}\left(\tau>t \mid \mathcal{F}_{t}\right)=\mathbb{P}\left(\tau>t \mid \mathcal{F}_{\infty}\right)$, a characterisation of immersion that we have recalled above. From our result, immersion holds since $\mathbb{F}$ is immersed in $\mathbb{F}^{\xi}$, hence in $\mathbb{F}^{\tau}$, due to the property that $\vartheta$ is an $\mathbb{F}$-stopping time. 
Let us also remark here the form of the dual optional projection of $A^{\tau}=\mathbb{1}_{\llbracket \tau, \infty \llbracket}$ in terms of the dual optional projections of $A^{\xi}=\mathbb{1}_{\llbracket \xi, \infty \llbracket}$ and $A^{\vartheta}=\mathbb{1}_{\llbracket \vartheta, \infty \llbracket}$. Let $H$ be an F-optional bounded process and recall that $A^{i}=\mathbb{1}_{\llbracket \tau_{i}, \infty \llbracket}$ for $i=1,2$. Then

$$
\begin{aligned}
\mathbb{E}\left[\int_{[0, \infty)} H_{s} d A_{s}^{1, o}\right] & =\mathbb{E}\left[H_{\tau_{1}} \mathbb{1}_{\left\{\tau_{1}<\infty\right\}}\right]=\mathbb{E}\left[H_{\xi} \mathbb{1}_{\{\xi<\infty\}} \mathbb{1}_{\{\xi<\vartheta\}}\right] \\
& =\mathbb{E}\left[H_{\xi} \mathbb{P}\left(\xi<\vartheta \mid \mathcal{F}_{\xi}\right) \mathbb{1}_{\{\xi<\infty\}}\right]=\mathbb{E}\left[H_{\xi} \gamma_{\xi} \mathbb{1}_{\{\xi<\infty\}}\right]
\end{aligned}
$$

where $\gamma$ is an $\mathbb{F}$-optional bounded process such that $\gamma_{\xi}=\mathbb{P}\left(\xi<\vartheta \mid \mathcal{F}_{\xi}\right)$. Finally we conclude that $A_{t}^{1, o}=\int_{[0, t]} \gamma_{s} d A_{s}^{\xi, o}$. In the same way we compute that $A_{t}^{2, o}=\int_{[0, t]} \kappa_{s} d A_{s}^{\vartheta, o}$ where $\kappa$ is another $\mathbb{F}$-optional process satisfying $\kappa_{\vartheta}=\mathbb{P}\left(\vartheta<\xi \mid \mathcal{F}_{\vartheta}\right)$. Then, by Proposition C.2 (b) we conclude that

$$
A_{t}^{\tau, o}=\int_{[0, t]} \gamma_{s} d A_{s}^{\xi, o}+\int_{[0, t]} \kappa_{s} d A_{s}^{\vartheta, o} .
$$

Note that $A^{\tau, p}$ can be expressed analogously.

\section{A Definitions of projections}

We collect here the definitions of the key tools we have used along the paper. Projections and dual projections onto the reference filtration $\mathbb{F}$ play an important role in the theory of enlargement of filtrations. First we recall the definition of optional and predictable projections, see [11, Theorems 5.1 and 5.2] and [16, p.264-265].

Definition A.1. Let $X$ be a measurable bounded (or positive) process. The optional projection of $X$ is the unique optional process ${ }^{\circ} X$ such that for every stopping time $T$, we have

$$
\mathbb{E}\left[X_{T} \mathbb{1}_{\{T<\infty\}} \mid \mathcal{F}_{T}\right]={ }^{o} X_{T} \mathbb{1}_{\{T<\infty\}} \quad \text { a.s. }
$$

The predictable projection of $X$ is the unique predictable process ${ }^{p} X$ such that for every predictable stopping time $T$ we have

$$
\mathbb{E}\left[X_{T} \mathbb{1}_{\{T<\infty\}} \mid \mathcal{F}_{T-}\right]={ }^{p} X_{T} \mathbb{1}_{\{T<\infty\}} \quad \text { a.s. }
$$

For definition of dual optional projection and dual predictable projection see [16, p.265], [25, Chapter 3 Section 5], [8, Chapter 6 Paragraph 73 p.148], [11, Sections 5.18, 5.19]. We point out that the convention we use here allows a jump at 0 , where for a finite variation process $V$ we assume that $V_{0-}=0$.

Definition A.2. (a) Let $V$ be a càdlàg pre-locally integrable variation process (not necessary adapted). The dual optional projection of $V$ is the unique optional process $V^{o}$ such that for every optional process $H$ we have

$$
\mathbb{E}\left[\int_{[0, \infty)} H_{s} d V_{s}\right]=\mathbb{E}\left[\int_{[0, \infty)} H_{s} d V_{s}^{o}\right] .
$$

In particular, $V_{0}^{o}=\mathbb{E}\left[V_{0} \mid \mathcal{F}_{0}\right]$.

(b) Let $V$ be a càdlàg locally integrable variation process (not necessary adapted). The dual predictable projection of $V$ is the unique predictable process $V^{p}$ such that for every predictable process $H$ we have

$$
\mathbb{E}\left[\int_{[0, \infty)} H_{s} d V_{s}\right]=\mathbb{E}\left[\int_{[0, \infty)} H_{s} d V_{s}^{p}\right] .
$$

In particular, $V_{0}^{p}=\mathbb{E}\left[V_{0} \mid \mathcal{F}_{0}\right]$. 


\section{B Summary of results on honest times}

For reader's convenience we gather complementary results on honest times. They can be found in [17] (see Lemma 5,1 and its proof there).

Proposition B.1. (a) A random time $\tau$ is an $\mathbb{F}$-honest time if and only if for every $t>0$ there exists an $\mathcal{F}_{t-}$-measurable random variable $\tau_{t}$ such that $\tau=\tau_{t}$ on $\{\tau<t\}$.

(b) A random time $\tau$ is an $\mathbb{F}$-honest time if and only if for every $t>0$ there exists an $\mathcal{F}_{t}$-measurable random variable $\tau_{t}$ such that $\tau=\tau_{t}$ on $\{\tau \leq t\}$.

Proof. Sufficiency of both conditions is straightforward.

Using the notation from Definition 3.1, we introduce the process $\alpha^{-}$as $\alpha_{t}^{-}=$ $\sup _{r \in Q, r<t} \tau_{r}$. This definition implies that $\alpha^{-}$is an increasing, left-continuous, adapted process such that $\alpha_{t}^{-}=\tau$ on $\{\tau<t\}$ thus the necessary condition in (a) is proven.

Let us denote by $\alpha$ the right-continuous version of $\alpha^{-}$, i.e., $\alpha_{t}=\alpha_{t+}^{-}$. Then, $\alpha$ is an increasing, càdlàg, adapted process such that $\alpha_{t}=\tau$ on $\{\tau \leq t\}$ and $\tau=\sup \left\{t: \alpha_{t}=t\right\}$ thus the necessary condition in (b) is proved.

Theorem B.2. Let $\tau$ be a random time. Then, the following conditions are equivalent:

(a) $\tau$ is an honest time;

(b) there exists an optional set $\Gamma$ such that $\tau(\omega)=\sup \{t:(\omega, t) \in \Gamma\}$ on $\{\tau<\infty\}$;

(c) $\widetilde{Z}_{\tau}=1$ a.s. on $\{\tau<\infty\}$;

(d) $\tau=\sup \left\{t: \widetilde{Z}_{t}=1\right\}$ a.s. on $\{\tau<\infty\}$.

Remark B.3. For progressive enlargement with an honest time, the hypothesis $\left(\mathcal{H}^{\prime}\right)$ is satisfied, and the following decomposition is given in [17, Theorem $(5,10)]$. Let $M$ be an $\mathbb{F}$-local martingale. Then, there exists an $\mathbb{F}^{\tau}$-local martingale $\widehat{M}$ such that:

$$
M_{t}=\widehat{M}_{t}+\int_{0}^{t \wedge \tau} \frac{1}{Z_{s-}} d\langle M, m\rangle_{s}-\int_{0}^{t} \mathbb{1}_{\{s>\tau\}} \frac{1}{1-Z_{s-}} d\langle M, m\rangle_{s} .
$$

For a thin honest time $\tau$, the two decomposition formulas, first given in Theorem 4.1 and second given in (B.1), coincide. It is enough to show that

$$
\int_{0}^{t} \mathbb{1}_{\{s>\tau\}} \frac{1}{1-Z_{s-}} d\langle X, 1-m\rangle_{s}=\sum_{n=1}^{\infty} \mathbb{1}_{C_{n}} \int_{0}^{t} \mathbb{1}_{\left\{s>T_{n}\right\}} \frac{1}{z_{s-}^{n}} d\left\langle X, z^{n}\right\rangle_{s} .
$$

This is a simple consequence of the set inclusion $\{\tau<s\} \cap\left\{\tau=T_{n}\right\} \subset\left\{T_{n}=\tau_{s} \leq s\right\}$ and Lemma C.4 (a):

$$
\begin{aligned}
\int_{0}^{t} \mathbb{1}_{\{s>\tau\}} \frac{1}{1-Z_{s-}} d\langle X, 1-m\rangle_{s} & =\sum_{n=1}^{\infty} \int_{0}^{t} \mathbb{1}_{\{s>\tau\} \cap\left\{\tau=T_{n}\right\}} \frac{1}{1-Z_{s-}} d\langle X, 1-m\rangle_{s} \\
& =\sum_{n=1}^{\infty} \int_{0}^{t} \mathbb{1}_{\{s>\tau\} \cap\left\{\tau=T_{n}\right\}} \frac{1}{z_{s-}^{n}} d\left\langle X, z^{n}\right\rangle_{s} \\
& =\sum_{n=1}^{\infty} \mathbb{1}_{C_{n}} \int_{0}^{t} \mathbb{1}_{\left\{s>T_{n}\right\}} \frac{1}{z_{s-}^{n}} d\left\langle X, z^{n}\right\rangle_{s} .
\end{aligned}
$$

EJP 26 (2021), paper 31. 


\section{Proofs and auxiliary results}

Proof of Lemma 1.5 (a) Define, for any $n \geq 0$, the $\mathbb{F}$-stopping time

$$
R^{n}:=\inf \left\{t \geq 0: z_{t}^{n}=0\right\}
$$

As $z^{n}$ is a positive càdlàg martingale, by [26, Proposition (3.4) p.70], it vanishes on $\llbracket R^{n}, \infty \llbracket$. Since $z^{n}$ is bounded, $z_{\infty}^{n}$ exists and:

$$
\left\{R^{n}<\infty\right\}=\left\{\inf _{t \geq 0} z_{t}^{n}=0\right\}=\left\{z_{\infty}^{n}=0\right\} .
$$

Moreover, the equality $0=\mathbb{E}\left[z_{\infty}^{n} \mathbb{1}_{\left\{z_{\infty}^{n}=0\right\}}\right]=\mathbb{E}\left[\mathbb{1}_{C_{n}} \mathbb{1}_{\left\{z_{\infty}^{n}=0\right\}}\right]$ implies that $C_{n} \cap\left\{z_{\infty}^{n}=0\right\}$ is a null set, so as well $C_{n} \cap\left\{\inf _{t} z_{t}^{n}=0\right\}$ is a null set. We obtain that $z^{n}>0$ and $z_{-}^{n}>0$ a.s. on $C_{n}$.

(b) The proof is based on monotone class theorem and we focus on a generator. The inclusion $\bigcap_{u>t} \mathcal{F}_{u} \vee \sigma\left(C_{n} \cap\left\{T_{n} \leq s\right\}, s \leq u, n \geq 1\right) \subset \mathcal{F}_{t}^{\tau}$ follows since $\tau$ and $T_{n}$ are $\mathbb{F}^{\tau}$-stopping times, therefore $\left\{\tau=T_{n}<\infty\right\} \in \mathcal{F}_{T_{n}}^{\tau}$ and $\left\{\tau=T_{n}<\infty\right\} \cap\left\{T_{n} \leq s\right\} \in \mathcal{F}_{s}^{\tau}$. The reverse inclusion is due to $\{\tau \leq s\}=\bigcup_{n=1}^{\infty} C_{n} \cap\left\{T_{n} \leq s\right\}$.

(c) By (a) and the monotone class theorem, for each $G \in \mathcal{F}_{t}^{\tau}$ there exists $F \in \mathcal{F}_{t}$ such that, for any $n \geq 1$,

$$
G \cap\left\{T_{n} \leq t\right\} \cap C_{n}=F \cap\left\{T_{n} \leq t\right\} \cap C_{n} .
$$

Then, using the fact that $T_{n}$ is an $\mathbb{F}$-stopping time, we have to show that

$$
\mathbb{E}\left[X \mathbb{1}_{\left\{t \geq T_{n}\right\} \cap C_{n}} z_{t}^{n} \mid \mathcal{F}_{t}^{\tau}\right]=\mathbb{1}_{\left\{t \geq T_{n}\right\} \cap C_{n}} \mathbb{E}\left[X \mathbb{1}_{\left\{t \geq T_{n}\right\} \cap C_{n}} \mid \mathcal{F}_{t}\right] .
$$

For any $G \in \mathcal{F}_{t}^{\tau}$, we choose $F \in \mathcal{F}_{t}$ satisfying (C.2), and we obtain

$$
\begin{aligned}
\mathbb{E}\left[X \mathbb{1}_{\left\{t \geq T_{n}\right\} \cap C_{n} \cap G} z_{t}^{n}\right] & =\mathbb{E}\left[X \mathbb{1}_{\left\{t \geq T_{n}\right\} \cap C_{n} \cap F} \mathbb{E}\left[\mathbb{1}_{C_{n}} \mid \mathcal{F}_{t}\right]\right] \\
& =\mathbb{E}\left[\mathbb{1}_{\left\{t \geq T_{n}\right\} \cap F} \mathbb{E}\left[\mathbb{1}_{C_{n}} \mid \mathcal{F}_{t}\right] \mathbb{E}\left[X \mathbb{1}_{C_{n}} \mid \mathcal{F}_{t}\right]\right] \\
& =\mathbb{E}\left[\mathbb{1}_{\left\{t \geq T_{n}\right\} \cap C_{n} \cap F} \mathbb{E}\left[X \mathbb{1}_{C_{n}} \mid \mathcal{F}_{t}\right]\right] \\
& =\mathbb{E}\left[\mathbb{1}_{\left\{t \geq T_{n}\right\} \cap C_{n} \cap G} \mathbb{E}\left[X \mathbb{1}_{C_{n}} \mid \mathcal{F}_{t}\right]\right]
\end{aligned}
$$

which ends the proof, taking into account that $z_{t}^{n}>0$ on $C_{n}$.

The next result gives the supermartingales $Z$ and $\widetilde{Z}$ of a thin time and their decompositions into an $\mathbb{F}$-martingale $m$ and the increasing process $A^{o}$ in terms of an exhausting sequence and martingale family of $\tau$. This is useful to check certain properties of thin (honest) times (we refer the reader to Sections 4.2 and 3).

Proposition C.1. Let $\tau$ be a thin time with exhausting sequence $\left(T_{n}\right)_{n \geq 0}$, partition $\left(C_{n}\right)_{n \geq 0}$ and martingale family $\left(z^{n}\right)_{n \geq 0}$. Then:

(a) $1-Z_{\tau}>0$ a.s. on $\{\tau<\infty\}$,

(b) $\widetilde{Z}_{t}=\sum_{n=0}^{\infty} \mathbb{1}_{\left\{t \leq T_{n}\right\}} z_{t}^{n}, Z_{t}=\sum_{n=0}^{\infty} \mathbb{1}_{\left\{t<T_{n}\right\}} z_{t}^{n}, A_{t}^{o}=\sum_{n=1}^{\infty} \mathbb{1}_{\left\{t \geq T_{n}\right\}} z_{T_{n}}^{n}$ and $m_{t}=$ $\sum_{n=0}^{\infty} z_{t \wedge T_{n}}^{n}$

Proof. (a) We have $Z_{\tau} \mathbb{1}_{\{\tau<\infty\}}=\sum_{n=1}^{\infty} \mathbb{1}_{C_{n}} Z_{T_{n}}$ and, on $\left\{T_{n}<\infty\right\}$, we have

$$
1-Z_{T_{n}}=\mathbb{P}\left(\tau \leq T_{n} \mid \mathcal{F}_{T_{n}}\right) \geq \mathbb{P}\left(\tau=T_{n} \mid \mathcal{F}_{T_{n}}\right)=z_{T_{n}}^{n}
$$

Combined with Lemma 1.5 (a), this implies that $1-Z_{\tau}>0$ a.s. on $\{\tau<\infty\}$.

(b) Deriving the form of $Z$ and $\widetilde{Z}$ is straightforward. To compute $A^{o}$, note that for any 


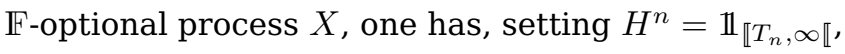

$$
\begin{aligned}
\mathbb{E}\left[\int_{[0, \infty)} X_{s} d A_{s}^{o}\right] & =\mathbb{E}\left[\int_{[0, \infty)} X_{s} d A_{s}\right]=\mathbb{E}\left[X_{\tau} \mathbb{1}_{\{\tau<\infty\}}\right]=\sum_{n=1}^{\infty} \mathbb{E}\left[X_{T_{n}} z_{T_{n}}^{n} \mathbb{1}_{\left\{T_{n}<\infty\right\}}\right] \\
& =\sum_{n=1}^{\infty} \mathbb{E}\left[\int_{[0, \infty)} X_{s} z_{s}^{n} d H_{s}^{n}\right]
\end{aligned}
$$

The form of $m$ follows by (1.1).

For $i \in\{1,2\}$, corresponding to the thin part $\tau_{1}$ and the thick part $\tau_{2}$ of a random time

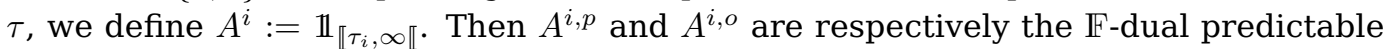
projection and the $\mathbb{F}$-dual optional projection of $A^{i}$. Let us denote by $Z^{i}$ and $\widetilde{Z}^{i}$ the supermartingales associated with $\tau_{i}$. Then, the following relations hold.

Proposition C.2. Let $\tau$ be a random time and $\left(\tau_{1}, \tau_{2}\right)$ its thin-thick decomposition.

(a) The supermartingales $Z$ and $\widetilde{Z}$ can be decomposed in terms of the supermartingales $Z^{1}, Z^{2}$ and $\widetilde{Z}^{1}, \widetilde{Z}^{2}$ as:

$$
Z=Z^{1}+Z^{2}-1 \text { and } \widetilde{Z}=\widetilde{Z}^{1}+\widetilde{Z}^{2}-1 .
$$

(b) The dual optional projection $A^{o}$ can be decomposed as

$$
A^{o}=A^{1, o}+A^{2, o}=A^{1, p}+A^{2, o} .
$$

(c) The dual predictable projection $A^{p}$ can be decomposed as

$$
A^{p}=A^{1, p}+A^{2, p}=A^{1, o}+A^{2, p} .
$$

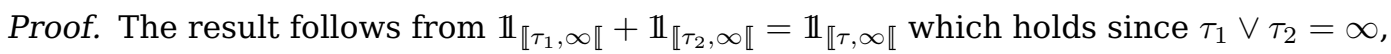
and by Theorem 2.2.

Lemma C.3. Let $\tau$ be a random time and $\left(\tau_{1}, \tau_{2}\right)$ its thin-thick decomposition. Let $\left(T_{n}\right)_{n \geq 0}$ be an $\mathbb{F}$-exhausting sequence, $\left(C_{n}\right)_{n \geq 0}$ an $\mathbb{F}$-partition and $\left(z^{n}\right)_{n \geq 0}$ an $\mathbb{F}$-martingale family of the $\mathbb{F}$-thin time $\tau_{1}$. Then, for any $t \geq 0$,

$$
\begin{aligned}
\mathbb{P}\left(C_{n} \mid \mathcal{F}_{t}^{\tau_{2}}\right) & =\mathbb{1}_{\left\{t<\tau_{2}\right\}} \frac{z_{t}^{n}}{Z_{t}^{2}} \text { for all } n \geq 0, \\
\mathbb{P}\left(\tau_{1}>t \mid \mathcal{F}_{t}^{\tau_{2}}\right) & =1-\mathbb{1}_{\left\{t<\tau_{2}\right\}} \frac{1-Z_{t}^{1}}{Z_{t}^{2}}, \\
\mathbb{P}\left(\tau_{2}>t \mid \mathcal{F}_{t}^{\tau_{1}}\right) & =1-\mathbb{1}_{\left\{t<\tau_{1}\right\}} \frac{1-Z_{t}^{2}}{Z_{t}^{1}} .
\end{aligned}
$$

Proof. Let us compute $\mathbb{P}\left(C_{n} \mid \mathcal{F}_{t}^{\tau_{2}}\right)$ on the two sets before $\tau_{2}$ and after $\tau_{2}$ separately as follows:

$$
\mathbb{P}\left(C_{n} \mid \mathcal{F}_{t}^{\tau_{2}}\right)=\mathbb{1}_{\left\{\tau_{2} \leq t\right\}} \mathbb{P}\left(C_{n} \mid \mathcal{F}_{t} \vee \sigma\left(\tau_{2}\right)\right)+\mathbb{1}_{\left\{t<\tau_{2}\right\}} \frac{\mathbb{P}\left(C_{n} \cap\left\{t<\tau_{2}\right\} \mid \mathcal{F}_{t}\right)}{\mathbb{P}\left(t<\tau_{2} \mid \mathcal{F}_{t}\right)}
$$

Then, taking into account that $C_{n}=\left\{\tau_{1}=T_{n}<\infty\right\}$ and $\tau_{1} \vee \tau_{2}=\infty$, one has on the one hand

$$
\mathbb{1}_{\left\{\tau_{2} \leq t\right\}} \mathbb{P}\left(C_{n} \mid \mathcal{F}_{t} \vee \sigma\left(\tau_{2}\right)\right)=\mathbb{P}\left(\tau_{1}=T_{n}<\infty, \tau_{2} \leq t \mid \mathcal{F}_{t} \vee \sigma\left(\tau_{2}\right)\right)=0,
$$

and, on the other hand,

$$
\mathbb{P}\left(C_{n} \cap\left\{t<\tau_{2}\right\} \mid \mathcal{F}_{t}\right)=\mathbb{P}\left(C_{n} \mid \mathcal{F}_{t}\right)=z_{t}^{n} .
$$


Therefore, the first equality holds.

By symmetry, we only prove the second identity and we skip the third one. Similarly as before we compute $\mathbb{P}\left(\tau_{1}>t \mid \mathcal{F}_{t}^{\tau_{2}}\right)$ on the two sets before $\tau_{2}$ and after $\tau_{2}$ separately as follows:

$$
\mathbb{P}\left(\tau_{1}>t \mid \mathcal{F}_{t}^{\tau_{2}}\right)=\mathbb{1}_{\left\{\tau_{2} \leq t\right\}} \mathbb{P}\left(\tau_{1}>t \mid \mathcal{F}_{t} \vee \sigma\left(\tau_{2}\right)\right)+\mathbb{1}_{\left\{t<\tau_{2}\right\}} \frac{\mathbb{P}\left(\tau_{2}>t, \tau_{1}>t \mid \mathcal{F}_{t}\right)}{\mathbb{P}\left(\tau_{2}>t \mid \mathcal{F}_{t}\right)} .
$$

Then, on the one hand, taking into account that $\tau_{1} \vee \tau_{2}=\infty$,

$$
\mathbb{1}_{\left\{\tau_{2} \leq t\right\}} \mathbb{P}\left(\tau_{1}>t \mid \mathcal{F}_{t} \vee \sigma\left(\tau_{2}\right)\right)=\mathbb{P}\left(\tau_{1}>t \geq \tau_{2} \mid \mathcal{F}_{t} \vee \sigma\left(\tau_{2}\right)\right)=\mathbb{P}\left(t \geq \tau_{2} \mid \mathcal{F}_{t} \vee \sigma\left(\tau_{2}\right)\right)=\mathbb{1}_{\left\{\tau_{2} \leq t\right\}},
$$

and, on the other hand, by $\tau_{1} \wedge \tau_{2}=\tau$ and Proposition C.2 (a),

$$
\mathbb{P}\left(\tau_{1}>t, \tau_{2}>t \mid \mathcal{F}_{t}\right)=\mathbb{P}\left(\tau>t \mid \mathcal{F}_{t}\right)=Z_{t}=Z_{t}^{1}+Z_{t}^{2}-1 .
$$

Therefore,

$$
\mathbb{P}\left(\tau_{1}>t \mid \mathcal{F}_{t}^{\tau_{2}}\right)=\mathbb{1}_{\left\{\tau_{1} \leq t\right\}}+\mathbb{1}_{\left\{t<\tau_{2}\right\}} \frac{Z_{t}^{1}+Z_{t}^{2}-1}{Z_{t}^{2}}
$$

and the result follows.

Lemma C.4. Let $\tau$ be a thin honest time, $\tau_{t}$ be associated with $\tau$ as in Definition 3.1 and $\left(T_{n}\right)_{n \geq 0}$ be an exhausting sequence of $\tau$. Then:

(a) on $\left\{T_{n}=\tau_{t}\right\}=\left\{T_{n}=\tau_{t} \leq t\right\}$, we have $z_{t}^{n}=1-Z_{t}, A_{t}^{o}=z_{T_{n}}^{n}$ and $1-m_{t}=z_{t}^{n}-z_{T_{n}}^{n}$ for each $n \geq 1$;

(b) on $\left\{T_{n}<t\right\}$ we have $z_{t}^{n}=\mathbb{1}_{\left\{\tau_{t}=T_{n}\right\}}\left(1-\widetilde{Z}_{t}\right)$ and $z_{t-}^{n}=\mathbb{1}_{\left\{\tau_{t}=T_{n}\right\}}\left(1-Z_{t-}\right)$ for each $n \geq 1$; in particular

$$
1-\widetilde{Z}_{t}=\sum_{n=1}^{\infty} \mathbb{1}_{\left\{\tau_{t}=T_{n}<t\right\}}\left(1-\widetilde{Z}_{t}\right) \quad \text { and } \quad 1-Z_{t-}=\sum_{n=1}^{\infty} \mathbb{1}_{\left\{\tau_{t}=T_{n}<t\right\}}\left(1-Z_{t-}\right) .
$$

Proof. (a) Using properties of $\tau_{t}$, we deduce that

$$
\begin{aligned}
\mathbb{1}_{\left\{T_{n}=\tau_{t}\right\}} z_{t}^{n} & =\mathbb{P}\left(T_{n}=\tau_{t} \leq t, \tau=T_{n}<\infty \mid \mathcal{F}_{t}\right) \\
& =\mathbb{P}\left(\tau \leq t, T_{n}=\tau_{t}=\tau \mid \mathcal{F}_{t}\right) \\
& =\mathbb{P}\left(\tau \leq t, T_{n}=\tau_{t} \mid \mathcal{F}_{t}\right) \\
& =\mathbb{1}_{\left\{T_{n}=\tau_{t}\right\}}\left(1-Z_{t}\right)
\end{aligned}
$$

where the first equality is due to $\tau_{t} \leq t$, the third one follows by $\tau_{t}=\tau$ on $\{\tau \leq t\}$ and the last one is true since $T_{n} \wedge t$ and $\tau_{t}$ are two $\mathcal{F}_{t}$-measurable random variables and

$$
\begin{aligned}
\left\{T_{n}=\tau_{t}\right\} & =\left\{T_{n}=\tau_{t}<t\right\} \cup\left\{T_{n}=\tau_{t}=t\right\} \\
& =\left\{\left\{T_{n} \wedge t=\tau_{t}\right\} \cap\left\{\tau_{t}<t\right\}\right\} \cup\left\{\left\{T_{n}=t\right\} \cap\left\{\tau_{t}=t\right\}\right\} .
\end{aligned}
$$

The dual optional projection of a thin time satisfies

$$
\mathbb{1}_{\left\{T_{n}=\tau_{t}\right\}} A_{t}^{o}=\sum_{k=1}^{\infty} \mathbb{1}_{\left\{T_{n}=\tau_{t}, T_{k} \leq t\right\}} z_{T_{k}}^{k}=\mathbb{1}_{\left\{T_{n}=\tau_{t}\right\}} z_{T_{n}}^{n},
$$

where the second equality is due to the fact that, for $n \neq k$, we have

$$
\begin{aligned}
\mathbb{1}_{\left\{T_{n}=\tau_{t}, T_{k} \leq t\right\}} z_{T_{k}}^{k} & =\mathbb{1}_{\left\{T_{n}=\tau_{t}\right\}} \mathbb{E}\left(\mathbb{1}_{\left\{\tau=T_{k} \leq t\right\}} \mid T_{k}\right) \\
& =\mathbb{1}_{\left\{T_{n}=\tau_{t}=T_{k}\right\}} \mathbb{E}\left(\mathbb{1}_{\left\{\tau=T_{k} \leq t\right\}} \mid T_{k}\right)=0
\end{aligned}
$$


since $T_{n}$ and $T_{k}$ have disjoint graphs and $\tau$ is an honest time. Combining the two previous points, we conclude that $1-m_{t}=1-Z_{t}-A_{t}^{o}=z_{t}^{n}-z_{T_{n}}^{n}$ on the set $\left\{T_{n}=\tau_{t}\right\}$.

(b) Again using properties of the random variable $\tau_{t}$, we derive

$$
\begin{aligned}
\mathbb{1}_{\left\{T_{n}<t\right\}} z_{t}^{n} & =\mathbb{P}\left(\tau=T_{n}=\tau_{t}<t \mid \mathcal{F}_{t}\right)=\mathbb{1}_{\left\{T_{n}=\tau_{t}<t\right\}}\left(1-\widetilde{Z}_{t}\right), \\
\mathbb{1}_{\left\{T_{n}<t\right\}} z_{t-}^{n} & =\mathbb{P}\left(\tau=T_{n}=\tau_{t}<t \mid \mathcal{F}_{t-}\right)=\mathbb{1}_{\left\{T_{n}=\tau_{t}<t\right\}}\left(1-Z_{t-}\right) .
\end{aligned}
$$

Then, Proposition C.1 (b) completes the proof.

\section{References}

[1] Aksamit, A., Choulli, T., Deng, J., and Jeanblanc, M. Arbitrages in a progressive enlargement setting. Arbitrage, Credit and Informational Risks, Peking University Series in Mathematics 6 (2014), 55-88. MR-3363797

[2] Aksamit, A., Choulli, T., Deng, J., and Jeanblanc, M. No-Arbitrage under a Class of Honest Times. Finance and Stochastics 22, 1 (2018), 127-159. MR-3738668

[3] Barlow, M. Study of a filtration expanded to include an honest time. Zeitschrift für Wahrscheinlichkeitstheorie und Verwandte Gebiete 44, 4 (1978), 307-323. MR-0509204

[4] Bielecki, T. R., Jeanblanc, M., and Rutkowski, M. Alternative approaches to credit risk modelling. Modèles aléatoires en finance mathématiques, Cours de Cimpa, Marrakech Maroc (2009), 67-159. MR-1869476

[5] Brémaud, P., and Yor, M. Changes of filtrations and of probability measures. Zeitschrift für Wahrscheinlichkeitstheorie und verwandte Gebiete 45, 4 (1978), 269-295. MR-0511775

[6] Coculescu, D. From the decompositions of a stopping time to risk premium decompositions. ESAIM: Proceedings and Surveys 56 (2017), 1-21. MR-3720061

[7] Dellacherie, C., and Meyer, P.-A. A propos du travail de Yor sur le grossissement des tribus. In Séminaire de Probabilités XII. Springer, 1978, pp. 70-77. MR-0519997

[8] Dellacherie, C., and Meyer, P.-A. Probabilités et potentiel: Chapitres 5 à 8 . Théorie des martingales. Hermann, 1980. MR-0727641

[9] Elliott, R. J., Jeanblanc, M., and Yor, M. On models of default risk. Mathematical Finance 10, 2 (2000), 179-195. MR-1802597

[10] Hannig, J. On filtrations related to purely discontinuous martingales. In Séminaire de Probabilités XXXVI. Springer, 2003, pp. 360-365. MR-1971597

[11] He, S.-w., Wang, J.-g., and Yan, J.-a. Semimartingale theory and stochastic calculus. Beijing: Science Press. Boca Raton, FL: CRC Press Inc., 1992. MR-1219534

[12] Jacod, J. Grossissement initial, hypothèse $\left(\mathcal{H}^{\prime}\right)$ et théorème de Girsanov. In Grossissements de filtrations: exemples et applications. Springer, 1985, pp. 15-35.

[13] Jacod, J., and Shiryaev, A. N. Limit theorems for stochastic processes, vol. 288. SpringerVerlag Berlin, 2003. MR-1943877

[14] Jacod, J., and Skorohod, A. V. Jumping filtrations and martingales with finite variation. In Séminaire de Probabilités XXVIII. Springer, 1994, pp. 21-35. MR-1329098

[15] Jeanblanc, M., and Le Cam, Y. Progressive enlargement of filtrations with initial times. Stochastic Processes and their Applications 119, 8 (2009), 2523-2543. MR-2532211

[16] Jeanblanc, M., Yor, M., and Chesney, M. Mathematical methods for financial markets. Springer, 2009. MR-2568861

[17] Jeulin, T. Semi-martingales et grossissement d'une filtration, vol. 833 of Lecture Notes in Mathematics. Springer, 1980. MR-0604176

[18] Jiao, Y., and Li, S. Modeling sovereign risks: from a hybrid model to the generalized density approach. Mathematical Finance 28, 1 (2018), 240-267. MR-3758923

[19] Kardaras, C. On the characterisation of honest times that avoid all stopping times. Stochastic Processes and their applications 124 (2014), 373-384. MR-3131298

[20] Kardaras, C., and Ruf, J. Filtration shrinkage, the structure of deflators, and failure of market completeness. Available at SSRN 3502510 (2019). MR-4152276 
Thin times and random times' decomposition

[21] Mansuy, R., and Yor, M. Random times and enlargements of filtrations in a Brownian setting. No. 1873 in Lecture notes in Mathematics. Springer, 2006. MR-2200733

[22] Meyer, P.-A. Sur un théorème de J. Jacod. In Séminaire de Probabilités XII. Springer, 1978, pp. 57-60. MR-0519995

[23] Nikeghbali, A. An essay on the general theory of stochastic processes. Probability Surveys 3 (2006), 345-412. MR-2280298

[24] Nikeghbali, A., and Yor, M. A definition and some characteristic properties of pseudo-stopping times. The Annals of Probability (2005), 1804-1824. MR-2165580

[25] Protter, P. Stochastic Integration and Differential Equations, 2.1 ed., vol. 21 of Stochastic Modelling and Applied Probability. Springer Verlag, 2004. MR-2273672

[26] Revuz, D., and Yor, M. Continuous martingales and Brownian motion, 3 ed. Springer, 1999. MR-1725357

[27] Song, S. Grossissement de filtration et problèmes connexes. PhD thesis, Université Paris VI (1987).

[28] Yoeurp, C. Théorème de Girsanov généralisé et grossissement d’une filtration. In Grossissements de filtrations: exemples et applications. Springer, 1985, pp. 172-196.

Acknowledgments. AA and MJ wish to acknowledge the generous financial supports of 'Chaire Markets in transition', French Banking Federation and ILB, Labex ANR 11LABX-0019. AA wishes to acknowledge the support of the European Research Council under the European Union's Seventh Framework Programme (FP7/2007-2013) / ERC grant agreement no. 335421. The research of TC is supported by the Natural Sciences and Engineering Research Council of Canada (NSERC), through Grant RGPIN 04987. We thank an anonymous referee for useful comments. 